\title{
An indigenous Saccharomyces uvarum population with high genetic diversity dominates uninoculated Chardonnay fermentations at a Canadian winery
}

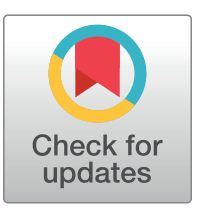

\section{OPEN ACCESS}

Citation: McCarthy GC, Morgan SC, Martiniuk JT, Newman BL, McCann SE, Measday V, et al. (2021) An indigenous Saccharomyces uvarum population with high genetic diversity dominates uninoculated Chardonnay fermentations at a Canadian winery. PLoS ONE 16(2): e0225615. https://doi.org/ 10.1371/journal.pone.0225615

Editor: Luca Cocolin, University of Torino, ITALY

Received: November 5, 2019

Accepted: December 29, 2020

Published: February 4, 2021

Peer Review History: PLOS recognizes the benefits of transparency in the peer review process; therefore, we enable the publication of all of the content of peer review and author responses alongside final, published articles. The editorial history of this article is available here: https://doi.org/10.1371/journal.pone.0225615

Copyright: @ $2021 \mathrm{McC}$ arthy et al. This is an open access article distributed under the terms of the Creative Commons Attribution License, which permits unrestricted use, distribution, and reproduction in any medium, provided the original author and source are credited.

Data Availability Statement: All raw data, $R$ scripts, software packages, versions, parameters, and primer sequences used in this study can be viewed at https://osf.io/j7rx8/. The QIIME2 artifact

\section{Garrett C. McCarthy ${ }^{1 \oplus}$, Sydney C. Morgan $\oplus^{10{ }^{*}}{ }^{*}$, Jonathan T. Martiniuk ${ }^{2}$, Brianne L. Newman ${ }^{1}$, Stephanie E. McCann ${ }^{1}$, Vivien Measday ${ }^{2}$, Daniel M. Durall ${ }^{1}$}

1 Department of Biology, Irfigving K. Barber School of Arts and Sciences, The University of British Columbia, Kelowna, British Columbia, Canada, 2 Wine Research Centre, Faculty of Land and Food Systems, The University of British Columbia, Vancouver, British Columbia, Canada

- These authors contributed equally to this work.

a Current address: Sanford Consortium for Regenerative Medicine, University of California San Diego, La Jolla, California, United States of America

*scmorgan@ health.ucsd.edu

\section{Abstract}

Saccharomyces cerevisiae is the primary yeast species responsible for most fermentations in winemaking. However, other yeasts, including Saccharomyces uvarum, have occasionally been found conducting commercial fermentations around the world. S. uvarum is typically associated with white wine fermentations in cool-climate wine regions, and has been identified as the dominant yeast in fermentations from France, Hungary, northern Italy, and, recently, Canada. However, little is known about how the origin and genetic diversity of the Canadian S. uvarum population relates to strains from other parts of the world. In this study, a highly diverse $S$. uvarum population was found dominating uninoculated commercial fermentations of Chardonnay grapes sourced from two different vineyards. Most of the strains identified were found to be genetically distinct from $S$. uvarum strains isolated globally. Of the 106 strains of $S$. uvarum identified in this study, four played a dominant role in the fermentations, with some strains predominating in the fermentations from one vineyard over the other. Furthermore, two of these dominant strains were previously identified as dominant strains in uninoculated Chardonnay fermentations at the same winery two years earlier, suggesting the presence of a winery-resident population of indigenous $S$. uvarum. This research provides valuable insight into the diversity and persistence of non-commercial $S$. uvarum strains in North America, and a stepping stone for future work into the enological potential of an alternative Saccharomyces yeast species.

\section{Introduction}

Modern winemaking often involves the inoculation of grape must with one or more commercial yeast strains, usually belonging to the dominant winemaking yeast species Saccharomyces 
can be viewed at https://view.qiime2.org. For raw Illumina sequence data, please visit https://qiita. ucsd.edu and search for Study 12837.

Funding: This work was supported by a Natural Sciences and Engineerig Research Council of Canada (NSERC) Discovery Grant (RGPIN-201604261) to V.M., and an NSERC Collaborative Research and Development Grant (CRDPJ 514045-17) to D.M.D. and V.M.

Competing interests: The authors have declared that no competing interests exist. cerevisiae. However, single-strain fermentations have been shown to produce less complex wines than fermentations conducted by multiple yeast species and strains [1-3]. Furthermore, the commercial S. cerevisiae strains used in these inoculated single-strain fermentations may be more aggressive than indigenous yeasts; indeed, commercial strains used previously at wineries have been identified entering and dominating uninoculated (spontaneous) fermentations in subsequent vintages [4-8]. The loss of indigenous yeast strains during the winemaking process can result in the reduction of regional character, because the local consortium of microorganisms can contribute to the expression of terroir in wine [3, 9]. In recent years, many winemakers have become interested in conducting uninoculated fermentations in an attempt to encourage a diversity of yeast species and strains to participate in alcoholic fermentation. Although vineyard-derived yeasts were originally thought to be the ones conducting uninoculated fermentations, a growing body of evidence has shown that uninoculated fermentations are actually conducted by winery-resident yeast strains $[4,7,10,11]$. These yeasts may be of commercial or indigenous origin, but have established themselves as residents of the winery environment, and are capable of entering and conducting fermentations over multiple vintages.

Although most uninoculated fermentations at commercial wineries are conducted by strains of S. cerevisiae $[4,7,10,11]$, some wineries contain established populations of Saccharomyces uvarum that are able to conduct and complete alcoholic fermentation [12-15]. S. uvarum belongs to the Saccharomyces sensu stricto clade, and is the furthest relative from $S$. cerevisiae within this clade $[16,17]$. The Holarctic S. uvarum population, which originated in the northern hemisphere [18-20], includes both natural S. uvarum strains isolated from the soil and bark of Quercus (oak) trees as well as industrial strains isolated from cider, beer, and wine fermentations. It is believed that the Holarctic population evolved alongside other Saccharomyces species, as $95 \%$ of Holarctic S. uvarum strains possess introgressed regions from Saccharomyces eubayanus, Saccharomyces kudriavzevii, and S. cerevisiae throughout their genome $[18,19]$. However, the history of $S$. uvarum research is difficult to trace, because $S$. uvarum has had many names, and has even shared names with now distinct species. In the past, S. uvarum has been referred to as Saccharomyces bayanus var. uvarum [13, 14, 21, 22] or simply Saccharomyces bayanus [23, 24]. To complicate matters, many commercial S. cerevisiae strains have been marketed incorrectly as strains of S. bayanus [25]. However, S. uvarum is now known to be a pure species, distinct from S. bayanus, which itself is a hybrid of the pure species $S$. uvarum and S. eubayanus $[16,19,26,27]$.

S. uvarum is a cryotolerant yeast usually found in association with white wine fermentations in cool-climate wine regions $[12,13,15,28]$, but has also been associated with cider production $[23,24]$ and some traditional fermentations $[29,30]$. During fermentation, S. uvarum produces lower levels of ethanol, acetic acid, and acetaldehyde, and higher levels of glycerol, succinic acid, malic acid, isoamyl alcohol, isobutanol, and ethyl acetate, as compared to $S$. cerevisiae [31-34]. Additionally, because of its ability to conduct fermentation at lower temperatures, S. uvarum may produce wines with more balanced aroma profiles [35]. However, few studies have been conducted to investigate the origins, genetic diversity, and enological potential of this yeast, and thus more research is needed on this topic.

The overall objective of this study was to identify the presence and genetic diversity of $S$. uvarum strains conducting uninoculated fermentations at a commercial winery in the Okanagan Valley wine region of British Columbia, Canada, and place this population within the context of global S. uvarum strains. Two years before this current study was conducted, a highly diverse, indigenous population of $S$. uvarum was identified at this same commercial winery [15]. We were interested in investigating the persistence of S. uvarum strains in the winery environment over multiple vintages. A secondary objective (addressed by sampling 
fermentations with grapes sourced from two different vineyards) was to investigate whether the geographical origin or chemistry of the grapes played a role in determining the fungal communities and S. uvarum populations present in the fermentations.

\section{Materials and methods}

\section{Experimental design and sampling}

This study was conducted in association with Mission Hill Family Estate Winery during the 2017 vintage. The fungal diversity and community composition of grapes from two different vineyards were followed from the vineyard into the winery and throughout alcoholic fermentation. Samples for high-throughput amplicon sequencing (Illumina MiSeq) were taken from grapes in the vineyard just prior to harvest, as well as at four stages of fermentation in the winery. Samples for Saccharomyces uvarum population analysis were taken at three stages of alcoholic fermentation.

Vineyard grape samples. Two Chardonnay vineyards managed by Mission Hill Family Estate Winery in the Okanagan Valley of British Columbia, Canada, were selected for this study (Vineyard 2 and Vineyard 8). Vineyard 2 is located approximately $120 \mathrm{~km}$ south of the winery, and Vineyard 8 is located approximately $90 \mathrm{~km}$ south of the winery; the two vineyards are approximately $30 \mathrm{~km}$ apart. The exact locations of these vineyards, along with the dates of grape sample collection, can be viewed in Table 1. Both vineyards had been herbicide-free since 2016 (2017 was the second herbicide-free vintage), and were transitioning from conventional to organic viticulture practices.

Each vineyard was divided into six sampling sections (achieved by dividing the number of rows in the vineyard by six), and was further sub-divided into potential sampling sites within each section. Each post in the vineyard was planted approximately 15 feet $(4.57 \mathrm{~m})$ apart, with five vines planted in between each post (within each panel). A potential sampling site was defined as three consecutive panels (equalling approximately 15 vines), and one sampling site was randomly selected within each sampling section, for a total of six samples per vineyard (S1 Fig). The following restrictions were placed on randomization: sampling occurred at least three posts inward from the edge of each vineyard, and at least two rows away from neighbouring vineyard blocks, to minimize the potential for contamination from nearby roads or other grape varietals. Sampling was performed by aseptically collecting one cluster from each vine in the sampling site, on both sides of the row, for a total of 30 clusters per sample (approximately $2-3 \mathrm{~kg}$ ). The 30 clusters from each sampling site were placed into a sterile bag (one bag per sampling site, six bags per vineyard) and transported on ice back to the laboratory at The University of British Columbia (Kelowna, BC, Canada) for same-day processing. Each bag containing grape clusters was then gently crushed and homogenized by hand for $10 \mathrm{~min}$, after which time $2 \mathrm{~mL}$ samples of the juice were collected and frozen at $-80^{\circ} \mathrm{C}$ to await further processing for high-throughput amplicon sequencing.

Winery samples. The winery portion of this study was conducted at Mission Hill Family Estate Winery, a large commercial winery in British Columbia, Canada that conducts both inoculated and uninoculated (spontaneous) fermentations of many different grape varietals and makes wines in many different styles. The Chardonnay grapes for this study were sourced

Table 1. Summary of vineyards used in this study.

\begin{tabular}{l|l|l|l|l|l}
\hline Vineyard name & Closest municipality & Latitude & Longitude & Sample collection & Date of commercial harvest \\
\hline Vineyard 2 & Osoyoos, BC & 49.000603 & -119.418712 & $2017-09-04$ & $2017-09-05$ \\
\hline Vineyard 8 & Oliver, BC & 49.221125 & -119.559834 & $2017-09-18$ & $2017-09-19$ \\
\hline
\end{tabular}

https://doi.org/10.1371/journal.pone.0225615.t001 
from two vineyards (Vineyard 2 and Vineyard 8), as described above. The must from each vineyard was crushed and pressed into large stainless steel tanks to undergo a settling period before being transferred into $285 \mathrm{~L}$ stainless steel barrels (La Garde, SML Stainless Steel, Québec, Canada). Must from Vineyard 2 spent two days in the settling tank, while must from Vineyard 8 spent eight days in the settling tank. Six stainless steel barrels were used in this experiment: three contained must exclusively from Vineyard 2, and three contained must exclusively from Vineyard 8. Because Vineyard 2 is located further south than Vineyard 8 (Table 1), the grapes from this vineyard ripened earlier, and were harvested before those from Vineyard 8, in order to achieve similar sugar concentrations at crush. Although the fermentations from each vineyard began at different times, they did overlap in the same cellar for 20 days during sampling. The cellar where the fermentations were conducted was maintained at $12^{\circ} \mathrm{C}$.

Samples were taken from the stainless steel barrels at four stages of uninoculated (spontaneous) alcoholic fermentation as defined by their sugar concentration (approximated by Brix level): cold settling ( $22^{\circ} \mathrm{Brix}$, prior to the start of AF), early ( $\left.14-18^{\circ} \mathrm{Brix}\right)$, mid $\left(6-10^{\circ} \mathrm{Brix}\right)$, and late $\left(2 \pm 0.1^{\circ} \mathrm{Brix}\right)$. To each barrel, $20 \mathrm{mg} / \mathrm{L}$ total sulfur dioxide was added in the form of potassium metabisulfite $\left(\mathrm{K}_{2} \mathrm{~S}_{2} \mathrm{O}_{5}\right)$ during the cold settling stage, 250 ppm Lallemand ${ }^{\mathbb{R}}$ Fermaid $\mathrm{K}$ complex yeast nutrient was added between the cold settling and early stages, and 100 ppm Laffort ${ }^{\mathbb{R}}$ THIAZOTE mineral nutrient was added between the early and mid stages. Samples were collected in sterile $50 \mathrm{~mL}$ centrifuge tubes and were transported on ice to the laboratory at The University of British Columbia (Kelowna, BC, Canada) for processing. Subsamples (2 $\mathrm{mL}$ ) were washed and frozen at $-80^{\circ} \mathrm{C}$ for high-throughput amplicon sequencing, while other subsamples were processed immediately for culture-dependent $S$. uvarum strain typing.

\section{Chemical analyses}

Chemical parameters for the grape must and wine were taken from the stainless steel barrels at the cold settling and late stages, respectively. Yeast assimilable nitrogen (YAN), titratable acidity, volatile acidity, malic acid, $\mathrm{pH}$, residual sugar, ethanol content, fructose, and glucose were measured using a WineScan ${ }^{\mathrm{TM}}$ wine analyzer (Foss, Hillerød, Denmark).

\section{Saccharomyces uvarum strain typing}

Colony isolation and DNA extraction. Samples from the early, mid, and late stages of fermentation were serially-diluted and plated onto YEPD media $(1 \%(\mathrm{w} / \mathrm{w})$ yeast extract $(\mathrm{BD}$

Bacto $^{\text {TM }}$, Sparks, MD, USA), 1\% (w/w) bacterial peptone (HiMedia Labs, Mumbai, India), 2\% (w/w) dextrose (Fisher Chemical, Fair Lawn, NJ, USA), 2\% (w/w) agar (VWR, Solon, OH, USA)), with the addition of $0.01 \%$ (v/v) chloramphenicol (Sigma-Aldrich, St. Louis, MO, USA) to prevent bacterial growth, and $0.015 \%(\mathrm{v} / \mathrm{v})$ biphenyl (Sigma-Aldrich, St. Louis, MO, USA) to prevent filamentous fungal growth [12,36-38]. Plates were incubated at $28^{\circ} \mathrm{C}$ for $48 \mathrm{~h}$ and stored at $4^{\circ} \mathrm{C}$. Plates containing 30-300 colonies were selected for colony isolation and DNA extraction. Grape samples from the vineyard and the cold settling stage samples were not analyzed for S. uvarum strains, because Saccharomyces yeasts are rarely found in must before the onset of alcoholic fermentation [39], and are present on healthy grapes in very low abundance compared to other microbes [40].

From the early, mid, and late stage plates, 47 single yeast colonies were isolated onto Wallerstein Nutrient media (WLN) (Sigma-Aldrich, St. Louis, MO, USA), a differential medium used to identify non-S. cerevisiae yeasts. Two controls were used to distinguish between S. cerevisiae and S. uvarum colonies: Lalvin ${ }^{\circledR}$ BA11 (Lallemand, Montreal, QC, Canada) and CBS 7001 (Westerdijk Fungal Biodiversity Institute, Utrecht, Netherlands), respectively. The WLN 
plates were incubated at $28^{\circ} \mathrm{C}$ for $48 \mathrm{~h}$, and then stored at $4^{\circ} \mathrm{C}$. Presumed S. cerevisiae isolates appeared cream-coloured, while presumed S. uvarum isolates appeared green (S2A Fig). In order to determine the colour of potential S. cerevisiae $\mathrm{x}$ S. uvarum hybrids on WLN plates, the following isolates were streaked onto a WLN plate and incubated at $28^{\circ} \mathrm{C}$ for $48 \mathrm{~h}$ : a pure $S$. cerevisiae strain (Lalvin ${ }^{\mathbb{R}}$ BA11), a pure S. uvarum strain (CBS 7001), and hybrid of S. cerevisiae $\mathrm{x}$ S. uvarum (Lalvin ${ }^{\circledR}$ S6U, formerly referred to as a S. cerevisiae $\mathrm{x}$ S. bayanus hybrid) (S2B Fig). DNA was extracted from each yeast isolate by performing a water DNA extraction, as described previously [7].

Multiplex PCR and fragment analysis. S. uvarum strain typing was performed as previously described [15] using a multiplex PCR reaction targeting 11 microsatellite loci that had been selected from two previous studies: L1, L2, L3, L4, L7, L8, L9 [41], NB1, NB4, NB8, and NB9 [28]. Briefly, multiplex PCR was performed on the extracted DNA from each presumed $S$. uvarum isolate and submitted to Fragment Analysis and DNA Sequencing Services at the University of British Columbia (Kelowna, BC, Canada) for fragment analysis on a 3130xl DNA sequencer (Applied Biosystems, Foster City, CA, USA). GeneMapper 4.0 software (Applied Biosystems, Foster City, CA, USA) was used to determine the fragment size at each locus, and the resulting multilocus genotype (MLG) of each isolate was compared to that of the others using Bruvo's genetic distance [42]. Yeast isolates obtained in this study were compared to a database containing 150 S. uvarum strains identified during the 2015 vintage at the same winery [15], as well as 12 international S. uvarum strains (Table 2).

Bruvo distance was calculated using an algorithm that takes into account stepwise mutations, making it appropriate for use with microsatellite data. Bruvo distance is calculated on experimental data and allows the user to collapse MLGs with slight differences in allele size into a single strain category, based on similarity at a threshold value from 0 to 1 . An applied threshold of 0 results in every unique MLG being classified as a different strain, and an applied threshold of 1 results in all the MLGs in a dataset being collapsed into a single strain. Bruvo distance was calculated in R (version 3.5.1) using the 'poppr' package (version 2.8.1) [43], and applying a genetic distance threshold of 0.3 for strain classification. A histogram of pairwise genetic distances was created in $\mathrm{R}$ (version 3.5.1) using the 'poppr' package (S3 Fig). Because the histogram was not bimodal, the largest gap between putative thresholds was determined visually as 0.3 [44]. Additionally, 0.3 was chosen as the cut-off threshold in order to remain consistent with the threshold used in a previous study of this same population [15]. This was particularly important because of the novelty of the high genetic diversity observed in this

Table 2. Names, geographical origins, and sources of international Saccharomyces uvarum strains used for comparison in this study.

\begin{tabular}{|c|c|c|}
\hline Name & Geographical Origin & Source \\
\hline CBS 395 & The Netherlands & \multirow[t]{3}{*}{ Centraalbureau voor Schimmelcultures (CBS) } \\
\hline CBS 7001 & Spain & \\
\hline CBS 8690 & Moldova & \\
\hline CBS 8696 & California (USA) & \multirow[t]{2}{*}{ Westerdijk Fungal Biodiversity Institute (Utrecht, The Netherlands) } \\
\hline CBS 8711 & France & \\
\hline PYCC 6860 & Hornby Island (Canada) & \multirow[t]{3}{*}{ Portuguese Yeast Culture Collection (PYCC) } \\
\hline PYCC 6861 & Hornby Island (Canada) & \\
\hline PYCC 6862 & Japan & \\
\hline PYCC 6871 & Portugal & \multirow[t]{3}{*}{ Universidade Nova de Lisboa (Caparica, Portugal) } \\
\hline PYCC 6901 & Oregon (USA) & \\
\hline PYCC 6902 & Missouri (USA) & \\
\hline Velluto BMV58 ${ }^{\mathrm{R}}$ & Spain & Commercial strain from Lallemand ${ }^{\circledR}$ \\
\hline
\end{tabular}

https://doi.org/10.1371/journal.pone.0225615.t002 
yeast population. Using a low cut-off threshold for distinguishing strains could overestimate this diversity, and artificially inflate the significance of our results; by using a conservative threshold, we are able to make more confident statements regarding the diversity of this population.

Isolates that only partially amplified were re-run and were subsequently excluded from analysis upon a second failure. Isolates that did not amplify were considered to be non-S. uvarum yeasts and were also excluded from analysis. Although 47 yeast isolates were originally selected from each sample, not all belonged to S. uvarum and some were presumed to be $S$. cerevisiae based on WLN. After strain identification, each sample was rarefied to $32 \mathrm{~S}$. uvarum isolates.

Presumed S. cerevisiae isolates were also strain-typed as described previously [15] in order to confirm their identities, but were not included in the analysis of this study.

RFLP analysis for hybrid investigation. Restriction fragment length polymorphism (RFLP) analysis was performed on 50 yeast isolates from this study, representing the most abundant 50 strains identified. A pure $S$. cerevisiae strain (Fermol ${ }^{\mathbb{R}}$ Mediterranée), a pure $S$. uvarum strain (CBS 7001), and a known S. cerevisiae x S. uvarum hybrid strain (Lalvin ${ }^{\mathbb{R}}$ S6U) were included in this analysis as reference strains. PCR-RFLP was performed via amplification of the ITS1 region of the rRNA gene using primers ITS1 and ITS4, followed by digestion using the restriction enzyme HaeIII (Product no. ER0151, Thermo Fisher, Waltham, MA, USA), as described previously [45]. The products were run on a $1.8 \%$ agarose gel and the resulting patterns of each of the 50 strains were compared to those of the reference strains (S4 Fig).

\section{High-throughput amplicon sequencing}

Sample treatment and DNA extraction. Samples for high-throughput amplicon sequencing (Illumina MiSeq) were taken from grapes in the vineyard (grapes), as well as from four stages of fermentation in the winery (cold settling, early, mid, and late).

Samples (previously frozen at $-80^{\circ} \mathrm{C}$ ) were thawed on ice, and then washed before total DNA was extracted following a modified protocol from a previously-published study [46]. Samples were pelleted by centrifugation at 13,200 rpm for $5 \mathrm{~min}$. The supernatant was discarded and the pellet was re-suspended in $1 \mathrm{~mL}$ chilled $1 \mathrm{x}$ phosphate-buffered saline (SigmaAldrich, St. Louis, MO, USA), then centrifuged again at 13,200 rpm for $5 \mathrm{~min}$. The supernatant was discarded and the pellet was re-suspended in $500 \mu \mathrm{L}$ of $50 \mathrm{mM}$ ethylenediaminetetraacetic acid (EDTA, pH 8.0) (Invitrogen, Grand Island, NY, USA). Samples were mixed by pipetting up and down five times, and the entire sample was transferred to a FastPrep tube (MP Biomedicals, Santa Ana, CA, USA) containing $200 \mathrm{mg}$ of $0.5 \mathrm{~mm}$ glass disruptor beads (Scientific Industries, Bohemia, NY, USA). The FastPrep tubes were placed into a Vortex-Genie 2 Digital bead beater (Scientific Industries, Bohemia, NY, USA) for two rounds of $2.5 \mathrm{~min}(30 / \mathrm{s})$, separated by $1 \mathrm{~min}$ on ice. Aliquots of $500 \mu \mathrm{L}$ Nuclei Lysis solution (Fisher, Hampton, VA, USA) were added to the FastPrep tubes and lysed in the bead beater for $1 \mathrm{~min}(30 / \mathrm{s})$. Samples were then incubated for $10 \mathrm{~min}$ at $95^{\circ} \mathrm{C}$ and then centrifuged at 13,200 rpm for $5 \mathrm{~min}$. To an autoclaved $2 \mathrm{~mL}$ microcentrifuge tube (VWR, Radnor, PA, USA), $500 \mu \mathrm{L}$ of the supernatant was added, followed by $250 \mu \mathrm{L}$ Protein Precipitation solution (Fisher, Hampton, VA, USA). Samples were then vortexed lightly and kept at room temperature $\left(22^{\circ} \mathrm{C}\right)$ for $15 \mathrm{~min}$. Samples were then centrifuged at 13,200 rpm for $5 \mathrm{~min}$, and $500 \mu \mathrm{L}$ of the supernatant was transferred to new $2 \mathrm{~mL}$ microcentrifuge tubes containing $75 \mu \mathrm{L} 20 \%$ (v/v) polyvinylpyrrolidone (PVP) solution (Sigma-Aldrich, St. Louis, MO, USA). Samples were pulse-vortexed for 10-20 sec and centrifuged at 13,200 rpm for $10 \mathrm{~min}$. Supernatant $(500 \mu \mathrm{L})$ was transferred to a new $2 \mathrm{~mL}$ microcentrifuge tube containing $300 \mu \mathrm{L}$ chilled 2-propanol (Sigma-Aldrich, St. Louis, MO, 
USA). Tubes were inverted to mix several times and left to sit at room temperature for $15 \mathrm{~min}$. Samples were then centrifuged at 13,200 rpm for $2 \mathrm{~min}$ and the supernatant was discarded. The pellet was re-suspended in $1 \mathrm{~mL}$ chilled $100 \%$ ethanol (Commercial Alcohols, Brampton, ON, Canada), centrifuged at 13,200 for $2 \mathrm{~min}$, and the entire supernatant was carefully discarded. Samples were left open in a biosafety cabinet for a maximum of 30 minutes to ensure complete evaporation of the ethanol. The samples were re-suspended in $50 \mu \mathrm{L}$ of $10 \mathrm{mM} \mathrm{TE}$ buffer (Invitrogen, Grand Island, NY, USA), and frozen at $-80^{\circ} \mathrm{C}$. A positive control, containing pure S. cerevisiae cells, and a negative control, containing only molecular-grade water, were also subjected to the same DNA extraction protocol, and were included during library preparation and Illumina sequencing.

Illumina MiSeq library preparation. Sample library preparation used a two-step PCR procedure consisting of 'amplicon' and 'index' PCR reactions, as described previously [47]. Amplicon PCR was performed by amplifying the ITS1 region of the rRNA gene using BITS and B58S3 primers [48] with CS1 and CS2 linker sequences, respectively. Index PCR primers contained Illumina MiSeq adapter sequences, unique eight nucleotide barcodes, 9-12 bp heterogeneity spacers, and CS1/CS2 linker sequences. After both PCR reactions, samples were submitted to the IBEST Genomics Resources Core at the University of Idaho (Moscow, ID, USA) for quantification, normalization, pooling, and sequencing. Paired-end sequencing ( $300 \mathrm{bp}$ ) was performed on an Illumina MiSeq Desktop Sequencer (Illumina Inc., San Diego, CA, USA).

Illumina MiSeq data processing. Illumina MiSeq data processing was performed using both R (version 3.5.1) and the open-source bioinformatics pipeline Quantitative Insights Into Microbial Ecology (QIIME2 version 2018.11) [49]. In R, sequences were denoised using the 'dada2' package (version 1.8) [50], as well as the 'ShortRead' (version 1.36.1) [51] and 'Biostrings' (version 2.46.0) packages. All forward and reverse primer sequences had been removed from the 5' end of the sequences by IBEST at the University of Idaho before being returned, but because some ITS sequences are likely to be shorter than $300 \mathrm{bp}$, it is possible that these sequences contain nucleotides from the opposite primer, which required removal before further processing using Cutadapt [52]. After all primer sequences had been removed, sequences were filtered and trimmed using the "filterandTrim" function in the 'dada2' package. Any sequence containing an $\mathrm{N}$ was removed, as well as any sequence shorter than $50 \mathrm{bp}$. The maximum number of expected errors allowed in any sequence was set to 2. Because the ITS1 region in fungi is highly variable $[53,54]$, trimming all sequences to the same length can reduce the diversity of the identified community and can even remove sequences with true lengths shorter than the specified truncation length. For this reason, sequences were not trimmed to a consistent length. Forward and reverse reads were merged using the "mergePairs" function, a sequence table was constructed with the "makeSequenceTable," and chimeras were removed with the "removeBimeraDenovo" function. The representative sequence table was converted to a Fasta file before being transferred from R to the QIIME2 pipeline to complete analysis.

In QIIME2, sequences underwent paired-end alignment using MAFFT [55], and a phylogenetic tree with a mid-point root was created using FastTree 2 [56]. Sequence variants were classified to the species level (if possible) using a dynamic (97-99\%) threshold classifier made with the UNITE (version 8.0) database [57]. Sequence variants that could not be classified to the order level or lower and those that appeared with a total frequency of $<100$ sequences were excluded from analysis. Samples were rarefied to 20,000 sequences before being exported from QIIME2 for statistical analysis and visualization. Two samples did not meet the applied threshold of 20,000 sequences, and were therefore removed from analysis: one sample was a cold settling sample from the Vineyard 8 fermentations, and the other was a grape sample from Vineyard 2. In the UNITE (version 8.0) database, S. uvarum is incorrectly classified as S. baya$n u s$, because in the past both species were considered synonymous. Based on our culture- 
dependent data, we are confident that sequences classified in the UNITE database as S. bayanus belong to $S$. uvarum, and we have accordingly re-named all our sequences identified as $S$. bayanus to S. uvarum.

\section{Statistical analysis}

All statistical analyses in this study, unless otherwise specified, were performed in $\mathrm{R}$ (version 3.5.1) using RStudio software, and all statistical tests assume a significance level of $\alpha=0.05$. The chemical parameters of the grape must and wine from each vineyard were statistically compared by performing a one-way analysis of variance (ANOVA), using the "aov" function. Each parameter was evaluated separately. Normality was assessed visually. Not all of the chemical parameters met the assumption of normal distribution, but as ANOVA are robust to departures from normality [58], no data transformations were performed. The assumption of homogeneity of variance was tested using the "leveneTest" function in the 'Rcmdr' package (version 2.5-1). Levene's test indicated no violation in the assumption of homogeneity of variance in any chemical parameter (S1 Table).

Rarefaction curves of both species richness (for the fungal community) and strain richness (for the S. uvarum population) were created using the "rarecurve" function in the 'vegan' package (version 2.5-1) (S5 Fig) [59]. The fungal community was rarefied to 20,000 sequences per sample; species richness reached a plateau at this sequencing depth for all the samples that were retained after rarefying (S5A Fig). The S. uvarum population did not reach terminal sampling depth before the rarefication point of 32 isolates per sample (S5B Fig); however, this number was chosen because it was the highest number of $S$. uvarum isolates that allowed every sample to be retained for analysis.

Simpson's Index of Diversity $(1-D)$ and Shannon's Diversity Index $(H)$ were calculated using the "diversity" function in the 'vegan' package (version 2.5-1) and reported \pm the standard error of the mean (SEM). Differences in diversity between the two vineyard treatments were assessed by performing a one-way ANOVA on grape samples using the "aov" function, and by performing one-way repeated-measures ANOVA on both the fungal communities and the $S$. uvarum populations throughout alcoholic fermentation, using the "Anova" function in the 'car' package (version 3.0-2). Grape samples were analyzed separately from the fermentation samples because they represent a different sample type, and cold settling samples were excluded from analysis because Vineyard 8 contained only two replicates at that stage. Residuals and histograms were plotted to test the assumptions of the model.

The relative abundance of fungal species and $S$. uvarum strains was visualized by creating stacked bar charts using GraphPad Prism (version 8.2.1) software (La Jolla, CA, USA). Differences in composition between vineyard treatments were assessed by performing permutational analysis of variance (PERMANOVA) tests using the "adonis" function (vegan package), and using Bray-Curtis dissimilarity matrices, which were calculated on untransformed abundance data using the "vegdist" function (vegan package). The assumption of homogeneity of multivariate group dispersions (PERMDISP) was analyzed using the "betadisper" and "permutest" functions (vegan package), using Bray-Curtis dissimilarity and calculating deviation from centroid. Test statistics ( $F$ values for PERMDISP and Pseudo- $F$ values for PERMANOVA) were calculated based on 999 permutations of raw data. No violation of the assumption of homogeneity of multivariate group dispersions was observed for the grape samples $(F(1,9)=0.65$, $p=0.51)$ or the $S$. uvarum population during alcoholic fermentation $(F(1,16)=1.9, p=0.19)$. The fungal community during alcoholic fermentation did violate this assumption $(F(1,16)=$ $10.4, p=0.001)$, but PERMANOVA tests are considered robust to unequal variances among treatments [60], so no data transformations were made. With regards to the fungal 
community, the grape samples were analyzed separately from the fermentation samples because they represent a different sample type, and the cold settling samples were excluded from analysis because Vineyard 8 contained only two biological replicates at that stage.

A principal coordinates analysis ( $\mathrm{PCoA}$ ) was generated using the "wcmdscale" and "ordihull" functions (vegan package), using Bray-Curtis dissimilarity, in order to visualise the spatial distribution of the S. uvarum population among samples and treatments. The design of this study involved repeated measures, meaning some data were not independent, which could potentially lead to an overestimation of treatment differences as a result of the PERMANOVA tests, which cannot account for repeated measures. Therefore, the PCoA ordination was used to visualize distances between samples of different treatments $[9,61]$. Finally, we note that because the fermentations from both vineyards did not occur simultaneously (Vineyard 8 was harvested 14 days after Vineyard 2), and because the Vineyard 8 must spent longer in the large settling tank, it is possible that the differences in fungal communities and S. uvarum populations observed in this study are not solely a result of differences in vineyard geography.

S. uvarum population structure was assessed on the 102 indigenous strains identified in this study (36 unique to 2017, 66 found in both 2015 and 2017) [15], as well as 12 international $S$. uvarum strains from ten geographic locations, isolated from winery and natural environments around the world (Table 2), for a total of 114 strains. Population structure was assessed by performing Bayesian clustering in InStruct [62], using the admixture model with a burn-in of 500,000 and a total run of 100,000 iterations with 5 chains per cluster $(K)$, from $K=1$ to $\mathrm{K}=12$. The 12 clusters were made up of the two vintages analyzed at this winery as well as the 10 geographic origins of the 12 international strains. The Deviance Information Criterion (DIC) method, outlined previously [63], was used to determine the optimal number of subpopulations, which was found to be $\mathrm{K}=11$. A plot of DIC at the last chain of 5 , according to $\mathrm{K}$ revealed an additional, minor minimum plateau at $K=5$, thus $K=5$ was also included and passed onto CLUMPP for alignment of the chains. Five InStruct chains at $\mathrm{K}=11$ and $\mathrm{K}=5$ were aligned using the LargeKGreedy algorithm in CLUMPP (version 1.1.2) [64], with 10,000 random input orders. The highest $\mathrm{H}$ value derived from the CLUMPP population alignment was 0.51 for $\mathrm{K}=11$ and 0.99 for $\mathrm{K}=5$. The higher $\mathrm{H}$ value for the $\mathrm{K}=5$ clusters suggested better CLUMPP-alignment of the InStruct output than for $\mathrm{K}=11$, although the DIC value recommended the optimal number of clusters was 11 . Therefore, inferred ancestry profiles were visualized for both $\mathrm{K}=11$ and $\mathrm{K}=5$ subpopulations. The CLUMPP-aligned ancestry profiles were visualized using DISTRUCT (version 1.1) [65], which provides a stacked bar plot for each strain, with strains (bars) partitioned into coloured segments that correspond to membership coefficients of inferred subpopulations. ObStruct [66] was used to determine significance of the InStruct-inferred population structure. GenAlEx (version 6.5) $[67,68]$ was used to calculate the Probability of Identity $\left(\mathrm{P}_{\mathrm{I}}\right)$ and fixation indices $\left(\mathrm{F}_{\mathrm{ST}}\right.$ and $\left.\mathrm{F}_{\mathrm{IS}}\right)$ for the S. uvarum population observed in this study, and to estimate heterozygosity.

An unrooted, neighbour-joining phylogenetic tree was generated to compare the genetic relatedness of the indigenous S. uvarum strains and the 12 international strains. The phylogenetic tree was generated using the 'ape' package (version 5.2) [69], while clustering of strains was accomplished by using the Bayesian clustering output of InStruct ( $\mathrm{K}=5$ clusters) for statistical grouping of subpopulations in the tree. A dominant ancestor was identified if the inferred coefficient was equal to or higher than 0.75 (75\%), based on the metric used previously for a similar population analysis [41]. Strains were coloured according to their dominant inferred ancestor. Bootstrap values were obtained using the "bruvo.boot" function in the 'poppr' package (version 2.8.1) [43], which randomly sampled loci 1000 times to recalculate the percent support of the tree success. Only branches with 50\% support or higher were indicated on the tree. 
Table 3. Chemical parameters of stainless steel barrel-fermented Chardonnay wines sourced from two vineyards. Samples were taken at the cold settling stage (prior to the start of alcoholic fermentation) and at the late stage (towards the end of alcoholic fermentation). Values are the mean \pm SEM ( $n=3$ barrels per vineyard treatment). An asterisk next to the chemical parameter indicates a significant difference $(p \leq 0.05)$ between the two vineyards. Each chemical parameter was evaluated separately within each stage.

\begin{tabular}{|c|c|c|c|}
\hline Stage & Chemical Parameter & Vineyard 2 & Vineyard 8 \\
\hline \multirow[t]{5}{*}{ Cold settling } & $\mathrm{pH}^{*}$ & $3.37 \pm 0.006$ & $3.31 \pm 0.003$ \\
\hline & Residual sugar $\left({ }^{\circ} \mathrm{Brix}\right)^{*}$ & $22.3 \pm 0.0$ & $22.0 \pm 0.03$ \\
\hline & Yeast assimilable nitrogen $(\mathrm{mg} / \mathrm{L})^{*}$ & $77.7 \pm 5.6$ & $110.7 \pm 3.9$ \\
\hline & Titratable acidity $(\mathrm{g} / \mathrm{L})^{*}$ & $5.07 \pm 0.03$ & $7.20 \pm 0.0$ \\
\hline & Malic acid $(\mathrm{g} / \mathrm{L})^{*}$ & $2.20 \pm 0.0$ & $3.47 \pm 0.03$ \\
\hline \multirow[t]{7}{*}{ Late } & $\mathrm{pH}$ & $3.37 \pm 0.01$ & $3.41 \pm 0.01$ \\
\hline & Titratable acidity $(\mathrm{g} / \mathrm{L})^{*}$ & $7.20 \pm 0.2$ & $7.83 \pm 0.03$ \\
\hline & Malic acid $(\mathrm{g} / \mathrm{L})^{*}$ & $1.97 \pm 0.03$ & $2.40 \pm 0.06$ \\
\hline & Volatile acidity $(\mathrm{g} / \mathrm{L})^{*}$ & $0.47 \pm 0.003$ & $0.38 \pm 0.001$ \\
\hline & Ethanol (\% v/v) & $11.7 \pm 0.1$ & $11.3 \pm 0.1$ \\
\hline & Glucose $(\mathrm{g} / \mathrm{L})$ & $1.07 \pm 0.1$ & $1.37 \pm 0.3$ \\
\hline & Fructose $(\mathrm{g} / \mathrm{L})$ & $28.9 \pm 1$ & $27.1 \pm 2$ \\
\hline
\end{tabular}

https://doi.org/10.1371/journal.pone.0225615.t003

All raw data and $\mathrm{R}$ scripts used in the preparation of this manuscript can be viewed at https://osf.io/j7rx8/.

\section{Results and discussion}

\section{Fermentation progression and wine chemistry}

The total time from harvest of the Chardonnay grapes to the end of alcoholic fermentation was similar for the must from both vineyards: the must from Vineyard 2 completed fermentation 40 days after harvest, and the must from Vineyard 8 completed fermentation 37 days after harvest. However, the time the must spent in the settling tank before being transferred to barrels for fermentation differed between the two vineyard treatments. Must from Vineyard 2 spent only two days in the large stainless steel settling tank, while must from Vineyard 8 spent eight days, due to a higher proportion of solid material that required a longer settling time.

Sugar concentration at crush was similar between the two vineyards (Table 3). Although a significant difference was found between the vineyards for sugar concentration $\left({ }^{\circ} \mathrm{Brix}\right)$, this is likely because of the low variation observed within each treatment. It is unlikely that a difference of $0.3^{\circ}$ Brix would have any biologically relevant effects on the microbial composition of the wine. The $\mathrm{pH}$ was higher in the must from Vineyard 2, while yeast assimilable nitrogen, titratable acidity, and malic acid were all significantly higher in the must from Vineyard 8.

By the late stage of alcoholic fermentation, there was no significant difference between the wines from the two vineyards in terms of $\mathrm{pH}$, ethanol content, or glucose and fructose concentration (Table 3). Very little glucose remained in the wine by the end of alcoholic fermentation $(<2 \mathrm{~g} / \mathrm{L})$, while close to $30 \mathrm{~g} / \mathrm{L}$ fructose remained unfermented.

By the late stage, titratable acidity and malic acid were still significantly higher in the wines from Vineyard 8 than the wines from Vineyard 2 (Table 3). However, the change in malic acid from the cold settling to the late stage was also much greater in the wines from Vineyard 8. In the wines from Vineyard 2, the malic acid concentration decreased from $2.20 \mathrm{~g} / \mathrm{L}$ to $1.97 \mathrm{~g} / \mathrm{L}$, a decrease of approximately $10 \%$. Meanwhile, in the wines from Vineyard 8, the malic acid concentration changed from $3.47 \mathrm{~g} / \mathrm{L}$ to $2.40 \mathrm{~g} / \mathrm{L}$, a decrease of approximately $30 \%$. This could suggest a more significant presence of malic acid-degrading bacteria in the must from Vineyard 8. Indeed, a previous study conducted at this same winery with grapes from Vineyard 8 
identified Tatumella spp. in barrels that did not receive $\mathrm{SO}_{2}$ [15]. These bacteria were thought to originate in the vineyard, as they were present in vineyard samples, but seemed to be sensitive to $\mathrm{SO}_{2}$; the bacteria were not able to persist in treatments that received $40 \mathrm{mg} / \mathrm{L} \mathrm{SO}_{2}$ at crush. When they did persist, however, the malic acid was almost completely degraded by the end of alcoholic fermentation. It is possible that the addition in this current study of only 20 $\mathrm{mg} / \mathrm{L} \mathrm{SO}_{2}$ allowed a portion of these bacteria to survive in the must and perform a partial degradation of malic acid. Unfortunately, we did not identify the bacterial community in this current study, so more research is needed to confirm this hypothesis.

Volatile acidity (estimated as acetic acid) was found to be significantly higher in the wines from Vineyard 2 (Table 3). However, neither vineyard produced wines with unacceptable levels of volatile acidity, and all the wines in this study contained volatile acidity levels that were below its sensory detection threshold of $0.7 \mathrm{~g} / \mathrm{L}$ [70].

\section{Fungal communities}

Fungal community diversity. Fungal species diversity was highest in the grape and cold settling samples for both vineyards, and decreased for the early, mid, and late stages of fermentation (Table 4). A decrease in the diversity of fungal taxa is expected at the onset of alcoholic fermentation, because most yeasts and fungi present on grapes are non-fermentative, and are either killed by the presence of ethanol or absence of oxygen, or out-competed for space and nutrients by the more dominant yeasts [39, 71]. Diversity did not change throughout the three alcoholic fermentation stages (early, mid, and late) within each vineyard treatment, but the wines from Vineyard 8 had a consistently higher diversity than the wines from Vineyard 2, regardless of the diversity index used.

Fungal community composition. In total, 194 fungal species were identified in this study, of which 11 achieved $\geq 10 \%$ relative abundance in at least two samples (S2 Table). These 194 species belonged to 19 different classes and 37 different orders; however, only four classes (Dothideomycetes, Eurotiomycetes, Leotiomycetes, Saccharomycetes), and six orders (Capnodiales, Dothideales, Pleosporales, Eurotiales, Erysiphales, Saccharomycetales), were represented in the top $90 \%$ of all identified sequences. For a list of all species identified in this study along with their taxonomic classifications, please visit https://osf.io/j7rx8/.

A PERMANOVA was performed to test the differences in community composition between grape (vineyard) samples, which were found to be significantly different $(F(1,9)=8.3$, $\left.\mathrm{R}^{2}=0.48, p=0.002\right)$. Interestingly, the fungal communities of the grape and cold settling samples within each vineyard treatment were also different from each other (Fig 1). The cold settling samples were taken after the grapes had been harvested, crushed, and processed, so at

Table 4. Fungal species diversity, measured as Simpson's Index of Diversity $(1-D)$ and Shannon's Diversity Index $(H)$, of stainless steel barrel-fermented Chardonnay sourced from two different vineyards. Diversity \pm SEM was measured from grape samples taken in the vineyard ( $n=5$ for Vineyard $2, n=6$ for Vineyard 8 ), as well as at four stages in the winery: cold settling ( $n=3$ for Vineyard 2, $n=2$ for Vineyard 8 ), early, mid, and late ( $n=3$ for both vineyards at all three fermentation stages). For each diversity index, a one-way repeated-measures ANOVA was performed to compare the differences between vineyards across the three fermentation stages (cold settling samples were not included because Vineyard 8 contained only two replicates). Grape samples were analyzed separately by performing one-way ANOVA, because they constituted a different sample type. The $p$-values for each index are indicated in the appropriate columns, and any significant differences $(p \leq 0.05)$ are in bold.

\begin{tabular}{|c|c|c|c|c|c|c|}
\hline \multirow[b]{2}{*}{ Sample } & \multicolumn{3}{|c|}{ Simpson's diversity $(1-D)$} & \multicolumn{3}{|c|}{ Shannon's diversity $(H)$} \\
\hline & Vineyard 2 & Vineyard 8 & $p=$ & Vineyard 2 & Vineyard 8 & $p=$ \\
\hline Grapes & $0.85 \pm 0.03$ & $0.69 \pm 0.05$ & 0.02 & $2.51 \pm 0.20$ & $0.69 \pm 0.13$ & 0.005 \\
\hline Cold settling & $0.64 \pm 0.03$ & $0.87 \pm 0.02$ & & $1.65 \pm 0.14$ & $2.29 \pm 0.15$ & \\
\hline Early & $0.06 \pm 0.01$ & $0.21 \pm 0.09$ & \multirow[t]{3}{*}{0.01} & $0.17 \pm 0.01$ & $0.38 \pm 0.14$ & \multirow[t]{3}{*}{0.006} \\
\hline Mid & $0.06 \pm 0.01$ & $0.31 \pm 0.04$ & & $0.14 \pm 0.02$ & $0.61 \pm 0.08$ & \\
\hline Late & $0.06 \pm 0.01$ & $0.30 \pm 0.10$ & & $0.15 \pm 0.02$ & $0.57 \pm 0.20$ & \\
\hline
\end{tabular}

https://doi.org/10.1371/journal.pone.0225615.t004 

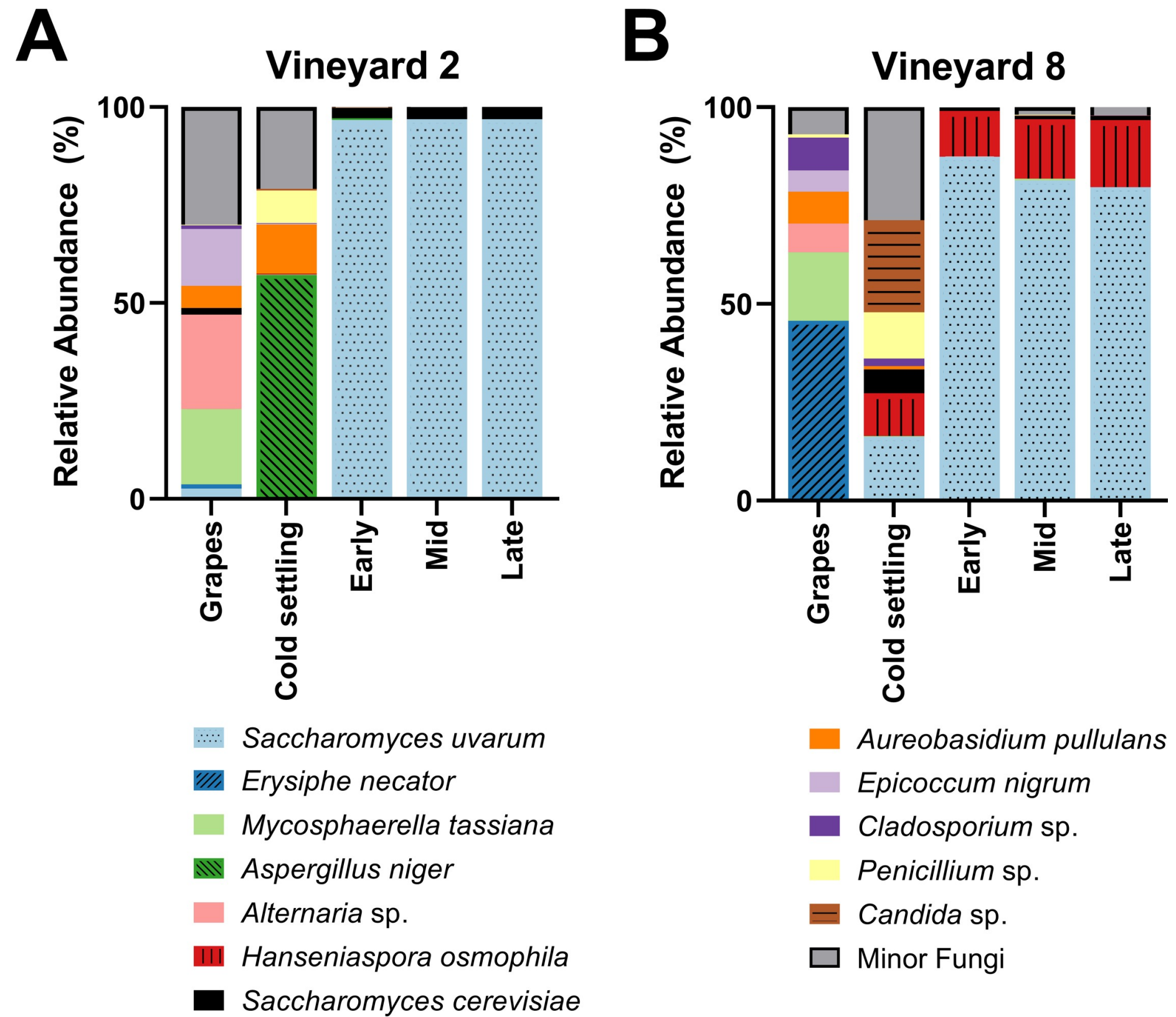

Fig 1. Fungal species abundance. Relative abundance of the dominant fungi present in grape samples taken in the vineyard (grapes), as well as at four stages of fermentation in the winery (cold settling, early, mid, and late), of Chardonnay sourced from (A) Vineyard 2 or (B) Vineyard 8 . Vineyard 2 grape sample values are the means of five replicates, and Vineyard 8 grape sample values are the means of 6 replicates. All winery fermentation stages have three reported replicates, with the exception of the cold settling stage from the Vineyard 8 fermentations, which contained two. Relative abundance was calculated from 20,000 sequences per sample. Any fungal taxa that did not achieve at least $10 \%$ relative abundance in at least two samples were grouped into the Minor Fungi category, with one exception: S. cerevisiae did not achieve $10 \%$ in any one sample, but because of its importance during alcoholic fermentation it has been included here. For variation among samples please see $\mathrm{S} 2$ Table.

least some of the differences observed between these stages can be attributed to contact with winery equipment. In the Vineyard 2 treatment, the most abundant fungi in the grape samples were Alternaria sp., Mycosphaerella tassiana, and Epicoccum nigrum, while the most abundant fungi in the cold settling samples were Aspergillus niger, Aureobasidium pullulans, and Penicillium sp. In the Vineyard 8 treatment, the most abundant fungi in the grape samples were 
Erysiphe necator and Mycosphaerella tassiana, while the most abundant fungi in the cold settling samples were Candida sp., Penicillium sp., S. uvarum, and Hanseniaspora osmophila.

A. pullulans is a ubiquitous environmental yeast-like fungus that is commonly associated with grapes and vineyards $[47,72,73]$, and was also the most common fungal species identified at the cold settling stage in must from Vineyard 8 two years prior to this current study [15]. $E$. nigrum is an endophytic fungus that has been previously identified in wine regions such as Italy, Portugal, and Spain [74-76]. Because both A. pullulans and E. nigrum are typically associated with vineyard environments, they have been proposed as potential biological control agents against grapevine pathogens [77]. Alternaria sp. are plant pathogens that are also commonly identified on grapes and in grape must $[47,78,79]$. M. tassiana, also known as Davidiella tassiana, is a common grape symbiont that has been previously isolated from vineyards $[15,47,80,81]$, and has been identified as the most abundant fungal species isolated on grapes in one study [79]. The presence of Aspergillus and Penicillium spp. in food, including grape must, has been observed previously $[15,47,82]$. These fungi have the potential to produce mycotoxins, which can be dangerous if consumed in large quantities [83, 84]. However, low levels of mycotoxins are common in commercial wines [84], and Canadian wines typically have a lower concentration of mycotoxins than wines produced elsewhere in the world [85]. Furthermore, the process of alcoholic fermentation has been shown to reduce the presence of mycotoxins, through enzymatic conversion to a less toxic form and/or the adsorption to lees and subsequent removal from the wine $[83,86]$. E. necator (also called Uncinula necator) is a grapevine pathogen responsible for powdery mildew, and is found in all regions of the world where grapes are grown [87]. The dominant presence of this fungus in the grape samples of Vineyard 8 is of potential concern, because the presence of even small amounts can have a negative effect on the overall sensory profile of the wine [88]. However, the grapes sampled in this study were not visibly infected with powdery mildew, and the presence of E. necator seems to have been eliminated by the time the cold settling sample was taken (Fig 1B). In the Vineyard 8 cold settling sample we observed the presence of yeasts with fermentative potential such as Candida sp., H. osmophila, and S. uvarum. These fermentative yeasts were not identified in the Vineyard 2 cold settling sample, likely because the must from Vineyard 8 spent an extended amount of time in the settling tank before being transferred to barrels (where the cold settling samples were taken), and therefore was more susceptible to early winery-resident yeast exposure. Although Candida and Hanseniaspora spp. have been found in association with grapes in the vineyard [89], in this current study it seems more likely that the origin of these yeasts is the winery equipment that the grapes came into contact with, because neither Candida nor Hanseniaspora spp. were identified in the grape samples from Vineyard 8 (S2 Table). The grape samples from Vineyard 2 had a greater presence of Saccharomyces spp. than the grape samples from Vineyard 8: S. cerevisiae was present at $1.61 \pm 1.3 \%$ and S. uvarum at $2.61 \pm 2.6 \%$ in Vineyard 2, and at $0.053 \pm 0.03 \%$ and $0.027 \pm 0.02 \%$, respectively, in Vineyard 8 (S2 Table). The abundance of Saccharomyces yeasts on healthy grapes in vineyards had been estimated at approximately 1/1000 yeast isolates [40], which is more in line with the results observed in Vineyard 8. The increased abundance of Saccharomyces yeasts in Vineyard 2 should be further investigated.

A PERMANOVA was performed to test the differences in fungal community composition throughout the three stages of alcoholic fermentation; a significant difference was observed between the two vineyard treatments $\left(F(1,16)=18.1, \mathrm{R}^{2}=0.53, p=0.001\right)$. S. uvarum dominated the early, mid, and late stages of alcoholic fermentation in both vineyard treatments. In the Vineyard 2 fermentations, S. uvarum made up $96.81 \pm 0.2 \%$ of the relative abundance of these three stages, and S. cerevisiae made up $2.91 \pm 0.2 \%$ (Fig 1A). In the Vineyard 8 fermentations, while $S$. uvarum still dominated with $82.88 \pm 3.5 \%$ of the relative abundance and $S$. 
cerevisiae was present at $0.89 \pm 0.2 \%$, a third yeast, $H$. osmophila, was also present, maintaining $14.56 \pm 3.2 \%$ relative abundance through to the end of alcoholic fermentation (Fig 1B). Originally, it was thought that Hanseniaspora spp. could not survive during the later stages of alcoholic fermentation due to low ethanol tolerance, because these yeasts could not be isolated from later stages using culture-dependent methods $[8,90,91]$. However, $H$. osmophila has been shown to have an ethanol tolerance of at least 9\% [92], and the advent of culture-independent identification techniques such as high-throughput amplicon sequencing have allowed for the identification of most microorganisms present in wine fermentations, including those in a viable but not culturable (VBNC) state. Indeed, other studies that have employed similar culture-independent techniques to ours have identified Hanseniaspora spp. through to the end of alcoholic fermentation [47, 71, 93-95]. H. osmophila has been characterized as a glucophilic yeast [92, 96, 97], and may have contributed to the alcoholic fermentation in the fermentations from Vineyard 8. However, it should be noted that the method of identifying the yeast community in this study involved analyzing DNA, not live cells, so it is possible that the DNA identified here as belonging to $H$. osmophila may not have come from living cells.

S. uvarum was also found dominating Chardonnay fermentations at this same winery two years previously [15], suggesting that this is a winery-resident population, capable of overwintering and entering fermentations year after year. Although it is possible that the S. uvarum population in this study was brought in from the vineyards with the grapes, we consider it more likely that the majority of these yeasts have established themselves as winery residents as opposed to vineyard residents. As mentioned above, Saccharomyces yeasts are typically rare on healthy grapes in the vineyard [40], and when Saccharomyces strains are identified in the vineyard, their presence is inconsistent between vintages [38, 98], and they are not necessarily the strains that conduct alcoholic fermentation in the winery $[99,100]$. The presence of wineryresident yeast populations and communities is well-established in the literature [72, 91, 101103], and these yeasts are capable of over-wintering in the winery and entering fermentations to which they have not been inoculated $[4,6,7,104]$. S. uvarum is usually found in association with white wine fermentations in cool-climate wine regions. It has been identified dominating such fermentations in wineries across Europe, including France [12, 13], Hungary [14, 34], Italy [105], and Slovakia [14]. S. uvarum is known to be cryotolerant [18, 27], explaining its preference for low-temperature fermentations and cool-climate wine regions. The cellar in which the fermentations from this study were conducted is temperature-controlled and kept at $12^{\circ} \mathrm{C}$. This temperature is within a desirable growth range for S. uvarum $[105,106]$, but is too low for S. cerevisiae to grow optimally $[34,105]$. Additionally, S. uvarum is a glucophilic yeast [33], and the residual fructose observed in the late stage of fermentation suggests that this yeast is less adept at fermenting fructose than $S$. cerevisiae. This result is supported by previous research [107].

To our knowledge, there is currently only one other winery (located in Alsace, France) that has been reported to have a local $S$. uvarum population dominating uninoculated fermentations across multiple vintages [12]. Interestingly, the Alsace cellar was also kept at $12^{\circ} \mathrm{C}$. It is possible that this temperature provides the optimal over-wintering conditions for S. uvarum, allowing it to out-compete S. cerevisiae, but more research is needed to investigate this.

\section{Saccharomyces uvarum strains}

Investigating the potential for S. uvarum hybrids. Because both S. cerevisiae and $S$. uvarum were identified in these fermentations, we acknowledge the potential for S. cerevisiae $\mathrm{x}$ S. uvarum hybrids in this community. Originally, 47 yeast colonies were isolated for strain identification; they were plated on WLN media in order to differentiate between presumed $S$. 


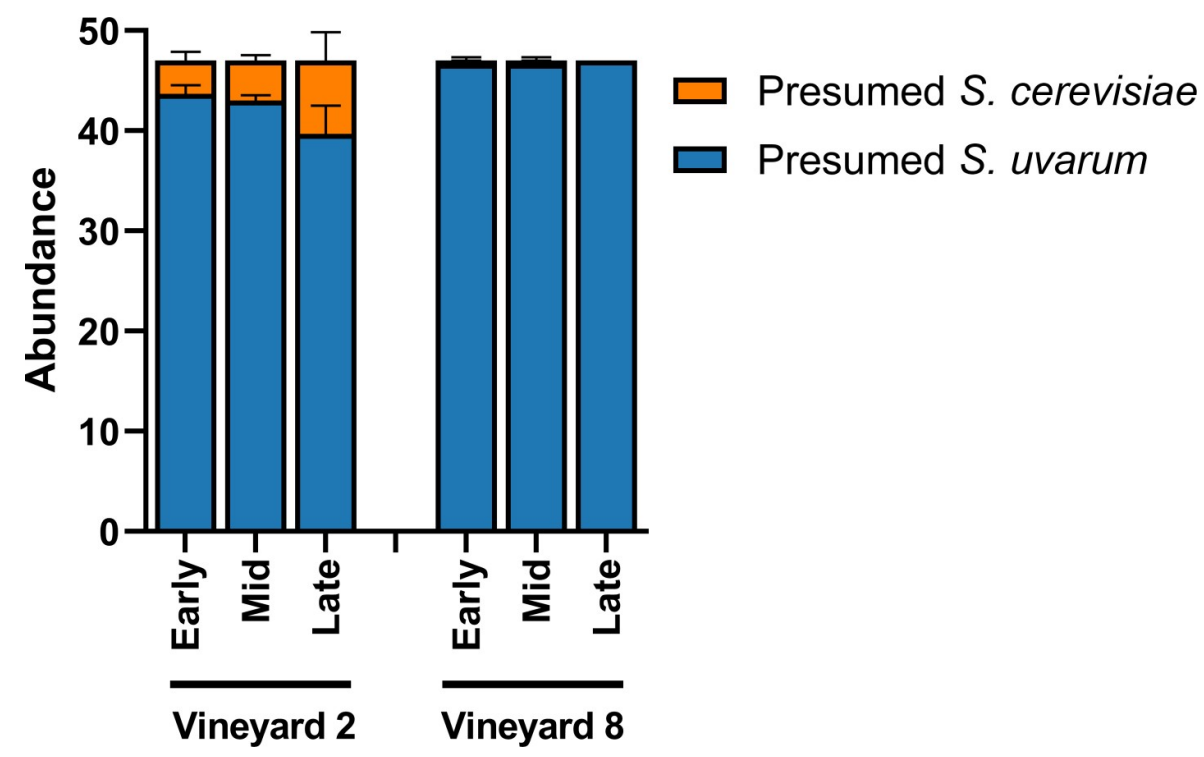

Fig 2. Relative abundance of presumed S. cerevisiae and S. uvarum. Relative abundance was determined based on phenotypical characteristics of presumed S. cerevisiae and presumed S. uvarum isolates present in three stages of fermentation (early, mid, and late) of Chardonnay sourced from two different vineyards $(n=3)$. Relative abundance was calculated from 47 yeast isolates per sample plated on Wallerstein Nutrient agar and presented \pm SEM.

https://doi.org/10.1371/journal.pone.0225615.g002

cerevisiae and presumed S. uvarum isolates (Fig 2). Additionally, in order to determine the potential colour of a hybrid strain on WLN plates, a pure S. cerevisiae strain (Lalvin BA11), a pure S. uvarum strain (CBS 7001), and a known S. cerevisiae x S. uvarum hybrid strain (Lalvin $\mathrm{S} 6 \mathrm{U}$ ) were all plated onto a WLN plate and incubated. The $S$. cerevisiae strain appeared as a cream-coloured colony, the $S$. uvarum strain appeared as a green colony, and the hybrid strain appeared as a cream-coloured colony (S2B Fig). Therefore, we conclude at least some hybrid strains may appear more similar to their S. cerevisiae parent when plated on WLN.

All but four of the presumed $S$. cerevisiae isolates were able to be strain-typed using primers that targeted S. cerevisiae microsatellite loci (for S. cerevisiae strain data see https://osf.io/j7rx8/ ). However, of the presumed S. uvarum isolates, a small proportion of the isolates only partially amplified when using primers that targeted $S$. uvarum microsatellite loci. These isolates were eventually removed from the analysis, and the $S$. uvarum population of each sample was rarefied to 32 S. uvarum strains, which represented the common denominator. The partial amplification of these isolates that were dropped from the analysis could suggest the presence of a small population of hybrid yeasts, as has been shown previously [41]. Another study conducted by Le Jeune et al. [108] characterized natural S. cerevisiae $\mathrm{x}$ S. uvarum hybrids where all loci amplified with primer sets from both species; however, only four $S$. uvarum microsatellite loci were targeted in that study.

To definitively determine if our S. uvarum strains were hybrids with S. cerevisiae, we conducted PCR-RFLP analysis on 50 isolates from this current study, representing the 50 most abundant strains identified. We amplified the ITS1 sequence of the rRNA gene, followed by digestion with the restriction enzyme HaeIII, which results in DNA fragments of different sizes for S. uvarum (180 bp, $230 \mathrm{bp}$, and $500 \mathrm{bp}$ ) and S. cerevisiae (180 bp, $230 \mathrm{bp}$, and $320 \mathrm{bp}$ ). All 50 of our isolates matched the restriction pattern of the reference $S$. uvarum strain (CBS 7001) (S4 Fig). The reference S. cerevisiae strain (Fermol Mediterranée) had a different restriction pattern, while the reference hybrid strain (Lalvin S6U) had a pattern that combined the patterns of the two pure species (S4 Fig). Therefore, we are confident that the strains included 
in the analysis of this study constitute pure S. uvarum strains, and not hybrids. Future studies specifically targeting $S$. uvarum hybrids will help determine if there is a significant hybrid population in the Okanagan wine region.

S. uvarum strain diversity. S. uvarum isolates were strain-typed using 11 microsatellite loci. Prior to strain-typing, presumed $S$. uvarum colonies were distinguished from presumed $S$. cerevisiae colonies by plating them on WLN media (S2 Fig). Similar to the Illumina fungal community results above, we observed an increase in the relative abundance of presumed $S$. cerevisiae isolates in the fermentations from Vineyard 2 (Fig 2).

S. uvarum strain diversity was similarly high in the fermentations from both vineyards (Table 5), and this high diversity was maintained throughout alcoholic fermentation. No significant difference in strain diversity was observed between the two vineyards. The study conducted in 2015 at this same winery also reported high S. uvarum strain diversity that was established early and was maintained throughout alcoholic fermentation [15]. This result has also been observed with regards to $S$. cerevisiae strain diversity in uninoculated commercial fermentations $[6,109]$.

\section{S. uvarum strain composition}

A total of 106 unique S. uvarum strains were identified across the six fermentations sampled in this study (S3 Table). Two years previously, 150 unique S. uvarum strains were identified in fermentations at this same winery [15]; 66 of these strains (44-62\% of all strains) were identified in both vintages. The previous study strain-typed 1,860 yeast isolates, in comparison to the 576 isolates strain-typed in this current study, so it is expected that fewer strains would be identified here. This large overlap in strains identified across vintages further suggests that most of these strains are winery residents: previous research investigating vineyard-derived $S$. cerevisiae strains found only a 9-13\% overlap in successive vintages [38]. Other studies conducted to investigate $S$. uvarum strain abundance have identified far fewer strains (a maximum of 89 unique strains isolated from grapes, wine, and other environments), although it should be noted that fewer isolates (up to 114) were strain-typed in those studies, potentially explaining this discrepancy $[12-14,22,28,41,105,110]$.

Of the 106 strains identified in this study, only four were able to achieve $\geq 10 \%$ relative abundance in at least two samples (Fig 3). The other 102 strains, termed 'minor strains,' accounted for $\sim 50 \%$ of the relative abundance in the Vineyard 2 fermentations, and $\sim 65 \%$ of the relative abundance in the Vineyard 8 fermentations. The two most abundant strains in both vineyard treatments ('2015 Strain 2' and '2015 Strain 3') were first identified as dominant strains at the same winery two years previously, during the 2015 vintage [15]. The reoccurrence of '2015 Strain 2' and '2015 Strain 3' after two years suggests that these strains have established themselves as persistent winery residents, capable of entering and dominating fermentations in multiple vintages. The other two dominant strains identified in these

Table 5. Saccharomyces uvarum strain diversity, measured as Simpson's Index of Diversity $(1-D)$ and Shannon's Diversity Index $(H)$, of stainless steel barrel-fermented Chardonnay sourced from two different vineyards. Diversity \pm SEM was measured from three stages of fermentation in the winery: early, mid, and late $(n=3)$. For each diversity index, a one-way repeated-measures ANOVA was performed to compare the differences between vineyards across all fermentation stages. The $p$-values for each index are indicated in the appropriate columns, and any significant differences $(p \leq 0.05)$ are in bold.

\begin{tabular}{|c|c|c|c|c|c|c|}
\hline \multirow[b]{2}{*}{ Sample } & \multicolumn{3}{|c|}{ Simpson's Index $(1-D)$} & \multicolumn{3}{|c|}{ Shannon's Index $(H)$} \\
\hline & Vineyard 2 & Vineyard 8 & $p=$ & Vineyard 2 & Vineyard 8 & $p=$ \\
\hline Early & $0.88 \pm 0.02$ & $0.92 \pm 0.009$ & \multirow[t]{3}{*}{0.19} & $2.46 \pm 0.16$ & $2.75 \pm 0.08$ & \multirow[t]{3}{*}{0.09} \\
\hline Mid & $0.89 \pm 0.01$ & $0.90 \pm 0.001$ & & $2.51 \pm 0.04$ & $2.59 \pm 0.07$ & \\
\hline Late & $0.85 \pm 0.03$ & $0.88 \pm 0.04$ & & $2.30 \pm 0.23$ & $2.57 \pm 0.26$ & \\
\hline
\end{tabular}

https://doi.org/10.1371/journal.pone.0225615.t005 


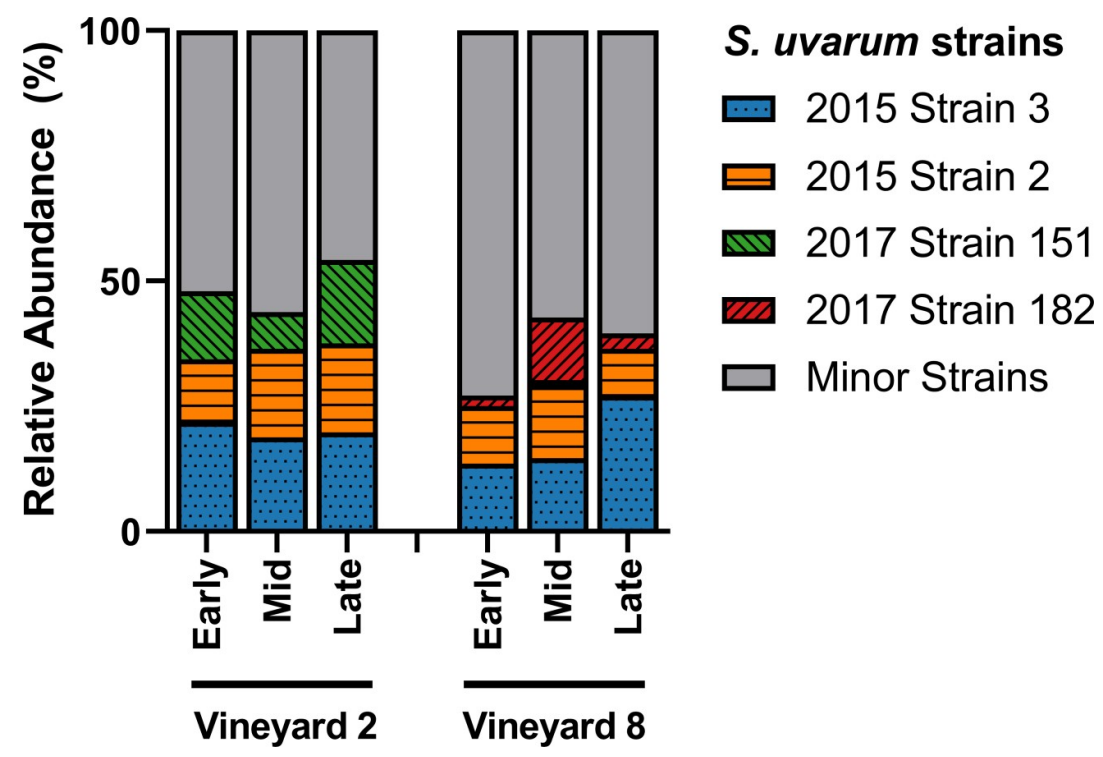

Fig 3. S. uvarum strain abundance. Relative abundance of the dominant $S$. uvarum strains present in three stages of fermentation (early, mid, and late) of Chardonnay sourced from two different vineyards $(n=3)$. Relative abundance was calculated from 32 S. uvarum yeast isolates per sample. Any strains that did not achieve $\geq 10 \%$ relative abundance in at least two samples were grouped into the Minor Strains category. For variation among samples please see S4 Table.

https://doi.org/10.1371/journal.pone.0225615.g003

fermentations ('2017 Strain 151' and '2017 Strain 182') were not isolated during the 2015 vintage, and were also not evenly distributed among the fermentations from the two vineyards (Fig 3). The '2017 Strain 151' represented 7-17\% of the relative abundance in the Vineyard 2 fermentations, and was only identified at $\sim 1 \%$ in a single sample in the Vineyard 8 fermentations. Meanwhile, the '2017 Strain 182' represented 2-12\% of the relative abundance in the Vineyard 8 fermentations, and was not identified at all in the Vineyard 2 fermentations. This difference in dominant strains could be attributed to a number of causes, including differences in acidity levels in the musts from the two different vineyards, or simply changes in the winery environment over time, since these fermentations did occur approximately two weeks apart. A significant correlation between grape must acidity and the dominance of specific S. cerevisiae strains has been previously observed [111], so it is understandable that a similar result might be expected with regards to $S$. uvarum strains. It is also possible that these two differentiallyabundant strains are predominantly vineyard-residents, with '2017 Strain 151' present in (or originating from) Vineyard 2, and '2017 Strain 182' present in (or originating from) Vineyard 8; this could explain their increased presence in one vineyard treatment over the other, but more research is needed in order to determine if this is the case.

A PERMANOVA was performed to test the differences in S. uvarum population composition throughout the three stages of alcoholic fermentation; a significant difference was observed between the two vineyard treatments $\left(F(1,16)=3.7, \mathrm{R}^{2}=0.19, p=0.001\right)$. A principal coordinates analysis (PCoA) was also generated in order to visualize the differences in $S$. uvarum strain composition between the fermentations from the two vineyards while including all 106 strains (Fig 4). The PCoA ordination showed a clear separation between samples taken from the two vineyards. This result highlights the importance of analyzing not only the diversity of a sample but also the composition. In this study, as in previous studies of a similar nature $[15,47]$, treatments have been found to have near-identical diversity values but completely distinct compositions. This is because composition considers not only the relative abundance of different strains, but also the identities of those strains, and can provide a more 
accurate summary of the differences observed among treatments. Previous research has identified that different strains of $S$. uvarum can produce wines with different sensory-active metabolite profiles, especially when fermented at lower temperatures [32,33], and it is therefore expected that different $S$. uvarum populations would contribute differently to the production of wine sensory attributes. However, this is still a new topic of investigation, and more research is needed in this area to determine whether the differences in secondary metabolite composition among wines fermented with different $S$. uvarum strains translate into detectable differences in the sensory profiles of the wines.

\section{S. uvarum genetic diversity}

Of the 106 strains identified in this study, 66 were also identified in the 2015 vintage at the same winery, including all four of the dominant strains from the 2015 vintage [15]. Additionally, we noted that 32 strains were unique to the Vineyard 2 fermentations, 40 strains were unique to the Vineyard 8 fermentations, and 34 strains were found in both the Vineyard 2 and Vineyard 8 fermentations. Although some minor strains were found to be unique to specific stages of fermentation, these strains were found in very low abundance: of the top 20 strains with the highest overall abundance, 18 were identified in all three stages of fermentation, suggesting that ethanol tolerance is not a major contributor to any differences in strain abundance observed here.

Four minor strains were found to bear genetic similarity to international strains (within a Bruvo distance of 0.3 ). Seven isolates from the Vineyard 2 fermentations were genetically similar to the commercial strain Velluto BMV58 ${ }^{\circledR}$, despite this strain not being sold in Canada at the time of this study. Additionally, three isolates were genetically similar to the previouslysequenced Spanish strain CBS 7001, one isolate was genetically similar to the French strain CBS 8711, and one isolate was genetically similar to strain PYCC 6860, which was isolated from oak trees on Hornby Island, in the same province of Canada as the winery from this study (although separated by hundreds of $\mathrm{km}$ ). Local strains bearing genetic relatedness to international strains has been previously observed; some S. uvarum strains isolated in New Zealand were also found to bear significant nucleotide similarity with CBS 7001 [28].

The probability that two unrelated individuals would have identical multilocus genotypes (MLGs) was calculated to be $P_{I}=6.7 \times 10^{-8}$. However, this probability does not take into account the Bruvo distance used in this study; individuals in this study could have slightly different MLGs and still be grouped into the same strain classification. Fixation indices were also calculated for this $S$. uvarum population, comparing the subpopulation identified in the Vineyard 2 fermentations with the subpopulation identified in the Vineyard 8 fermentations. The proportion of genetic variance contained within each vineyard treatment subpopulation relative to the entire population $\left(\mathrm{F}_{\mathrm{ST}}\right)$ was calculated to be $0.014 \pm 0.003$, indicating that the two subpopulations share a high degree of genetic material and suggesting a high level of interpopulation breeding. The inbreeding coefficient $\left(\mathrm{F}_{\mathrm{IS}}\right)$ was calculated to be $0.74 \pm 0.04$, indicating a considerable degree of inbreeding.

The occurrence of heterozygosity in this study was higher than was observed at the same winery two years previously: $51.9 \%$ of the strains in this study contained at least one heterozygous locus, as compared to $42.7 \%$ in 2015 [15]. This is the highest incidence of heterozygosity observed in an S. uvarum population to-date: previous studies have found heterozygous loci in $28.8 \%$ [41], 23.1\% [28], and 0\% [22] of S. uvarum strains isolated from grapes and wine. However, these studies did contain fewer isolates and analyzed fewer hypervariable microsatellite loci, which may explain some of the differences observed. The L9 microsatellite locus was the most variable by far, containing 18 different alleles in this study. The second most variable 


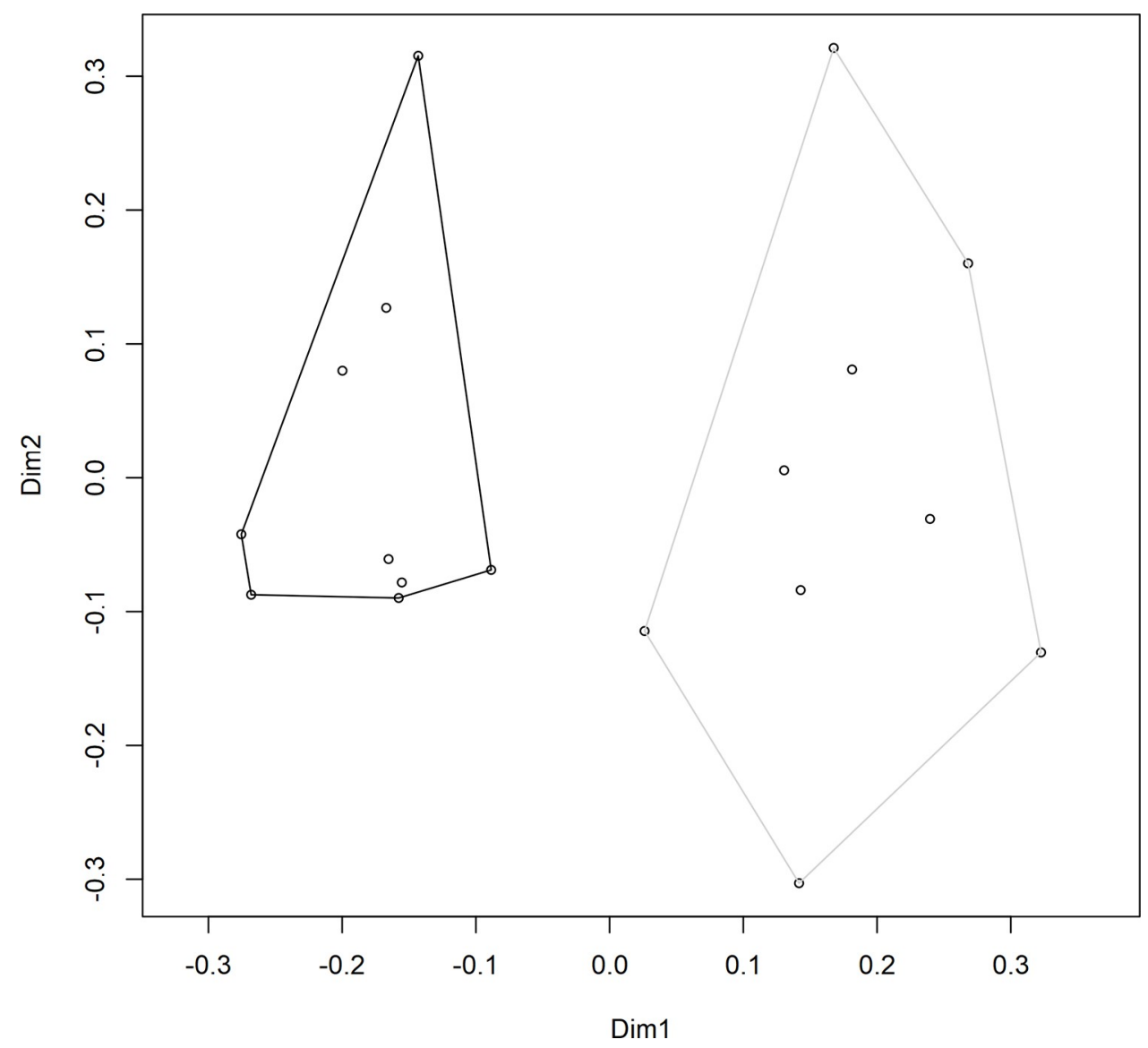

Fig 4. S. uvarum strain composition. Principal coordinates analysis (PCoA) ordination of the S. uvarum strain composition in Chardonnay wines sourced from two vineyards: Vineyard 2 (black) and Vineyard 8 (grey). Individual data points represent the composition of $S$. uvarum strains in a single sample (based on 32 yeast isolates per sample). Samples were taken at three stages of alcoholic fermentation, and each vineyard treatment contained three biological replicates, for a total of nine samples per vineyard. Dimension 1 (Dim1) explains $20.98 \%$ of variance, and Dimension 2 (Dim2) explains $13.07 \%$ of variance.

https://doi.org/10.1371/journal.pone.0225615.g004

locus was L2, containing eight different alleles. The other loci contained either five alleles (L7 and L8), four alleles (L1, L3, L4, and NB9), or three alleles (NB1, NB4, and NB8). While the rate of heterozygosity was higher in this population than has been previously observed, the observed heterozygosity rate for each locus was between 2 and 16 times lower than the unbiased expected heterozygosity rate for each locus, suggesting that this population still has a high selfing rate (for supporting data, visit https://osf.io/j7rx8/).

Population structure was assessed by performing Bayesian clustering on the 102 indigenous S. uvarum strains identified in the 2017 winery fermentations, as compared to 12 international strains (Table 2), using InStruct [62]. This method, which assigns strains membership coefficients to different inferred ancestors and takes into account inbreeding rates, has been previously used to assess the structure of both $S$. cerevisiae $[9,38,112,113]$ and S. uvarum [41] populations. Both $\mathrm{K}=11$ and $\mathrm{K}=5$ clusters were identified depending on the analysis method, and both were plotted to visualize the inferred ancestry of each strain using DISTRUCT (Fig 5).

A dominant inferred ancestor was identified if the inferred coefficient was equal to or higher than 0.75 (75\%) of the total ancestry; a similar metric has been used previously to infer dominant ancestry in S. uvarum populations [41]. For the $\mathrm{K}=11$ analysis (Fig 5B), no strain had 


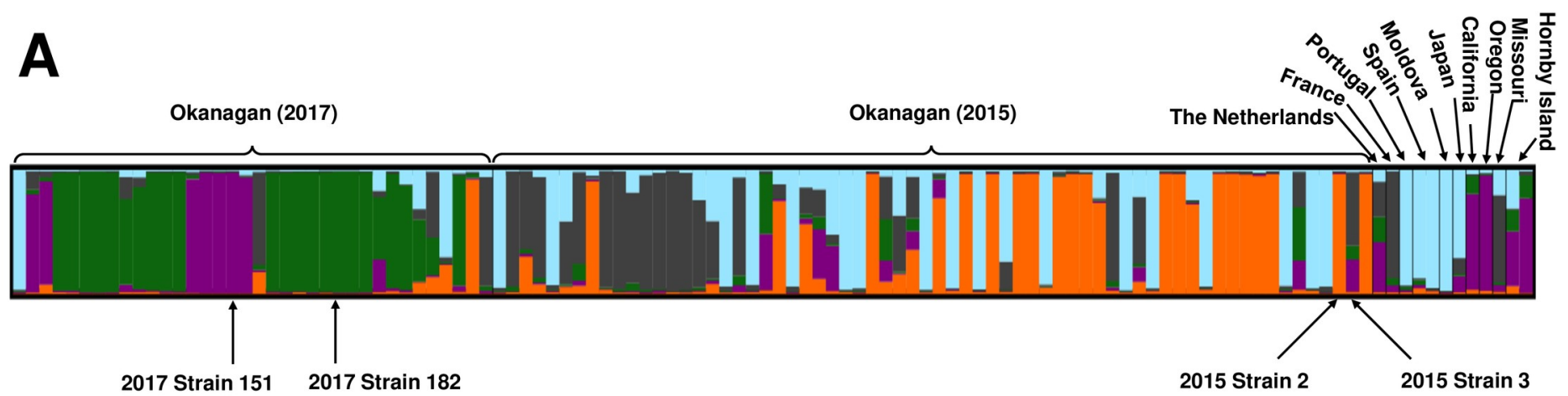

\section{Inferred ancestry:}
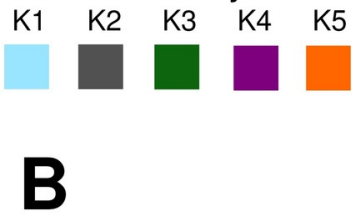

Okanagan (2017)

Okanagan (2015)

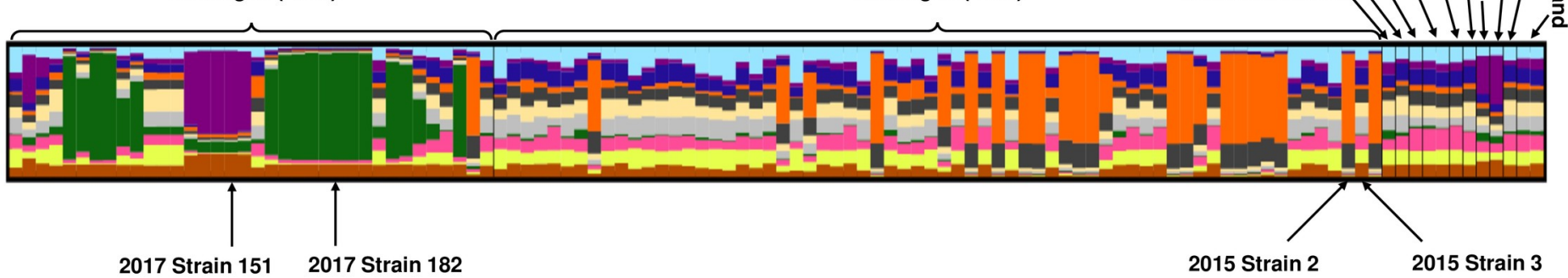

\section{Inferred ancestry:}

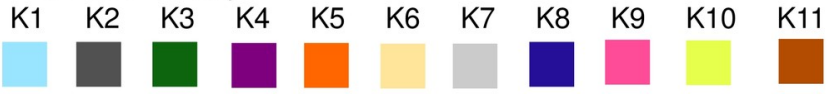

Fig 5. DISTRUCT plots of $S$. uvarum population structure by inferred ancestry. Each column represents a single $S$. uvarum strain, and different colours correspond to different inferred ancestors: (A) K1 to K5 inferred ancestry clusters, and (B) K1 to K11 inferred ancestry clusters. Strains included in this analysis include 12

international strains, as well as 102 indigenous strains isolated at an Okanagan winery during the 2017 vintage. Strains in the Okanagan (2015) section were originally identified at the same winery during the 2015 vintage, and were isolated again in 2017. Strains in the Okanagan (2017) section were unique to the 2017 vintage. The four dominant strains identified in this study are indicated.

https://doi.org/10.1371/journal.pone.0225615.g005

a single ancestor, and most strains were not dominated by any one subpopulation; instead, the strains appeared to have several ancestors, suggesting significant admixture between subpopulations. For the $\mathrm{K}=5$ analysis, most of the strains contained one dominant ancestor, although all strains contained some contribution from multiple inferred ancestors (Fig 5A). Many of the Okanagan strains identified in this study had a dominant inferred ancestor that was distinct from the international strains. Furthermore, there was a stark distinction in inferred ancestry profiles between the strains originally identified in 2015 and those identified for the first time in 2017, suggesting a strong vintage-to-vintage variability in at least a portion of the $S$. uvarum population. Indeed, an analysis using ObStruct [66] found that both the geographic origin and the vintage of origin (in the case of the Okanagan strains identified in this study) influenced population structure $\left(\mathrm{R}^{2}=0.28, p<0.001\right)$; interestingly the output of the ObStruct analysis also suggested that the predefined population of Okanagan S. uvarum strains identified for the first time in 2017 was the greatest driver of population structure (https://osf.io/j7rx8/).

Twenty of the strains originally identified in 2015 had K5 (orange) as the dominant inferred ancestor; none of the international strains had this structure, and only one of the 2017 strains 
had K5 (orange) as their dominant inferred ancestor (Fig 5A). Additionally, 10 of the 2015 strains had K2 (grey) as the dominant inferred ancestor, which was the case for just one 2017 strain and one international strain (CBS 8711 from France). For the strains unique to the 2017 vintage, more than half had $\mathrm{K} 3$ (green) as the dominant inferred ancestor; no international strains nor any 2015 strains had K3 (green) as a dominant inferred ancestor, making this result unique and specific to the strains from that vintage. Indeed, $\mathrm{K} 3$ (green) comprises on average less than $5 \%$ of the ancestry profiles of the international and 2015 strains. Additionally, seven of the 2017 strains had K4 (purple) as a dominant inferred ancestor. While none of the 2015 strains had this result, three international strains also had K4 (purple) as their dominant inferred ancestor, all from the west coast of North America: CBS 8696 (California), PYCC 6901 (Oregon), and PYCC 6861 (Hornby Island, British Columbia, Canada). Finally, a number of strains, both of international original and from this study, had K1 (blue) as the dominant inferred ancestor: these included one strain from 2017, 17 strains from 2015, and four international strains, from Portugal (PYCC 6871), Spain (CBS 7001 and BMV58), and Moldova (CBS 8690). The four dominant strains from this study all had different inferred ancestry. '2017 Strain 151' had K4 (purple) as its dominant inferred ancestor, '2017 Strain 182' had K3 (green) as its dominant inferred ancestor, and ' 2015 Strain 2' had K5 (orange) as its dominant inferred ancestor; for each of these strains, the dominant inferred ancestor represented almost $100 \%$ of the ancestry profile (coefficients $0.979,0.976$, and 0.971 , respectively). Contrastingly, '2015 Strain 3' did not contain a dominant inferred ancestor, and its inferred ancestry profile was split between K2 (grey), K3 (green), and K4 (purple) (coefficients 0.596, 0.110, and 0.260, respectively). The strain with the closest inferred ancestry profile to '2015 Strain 3' was strain CBS 395 from The Netherlands (coefficients K2 $=0.284, \mathrm{~K} 3=0.205, \mathrm{~K} 4=0.402$.

The dominant strains from this study had a similar structure using both the $\mathrm{K}=5$ and the $\mathrm{K}=11$ clustering methods. In both cases, '2017 Strain 151' had $\mathrm{K}=4$ (purple) as a majority ancestor, '2017 Strain 182' had a K = 3 (green) as a majority ancestor, and '2015 Strain 2' had $\mathrm{K}=5$ (orange) as a majority ancestor, and ' 2015 Strain 3' comprised multiple inferred ancestors, with none dominating.

A number of the Okanagan strains identified in this study (including one of the dominant strains), along with the west coast international strains from California, Oregon, and Hornby Island, contained K4 (purple) as a dominant or significant inferred ancestor. This suggests that these strains may have a shared geographical origin. Aside from these west coast strains, most of the Okanagan strains from both vintages had different majority inferred ancestors from the international strains. In particular, 41 of the 102 Okanagan strains had a K3 (green) or K5 (orange) dominant inferred ancestor, neither of which appeared in significant proportions in the inferred ancestry profiles of the international strains (Fig 5A). This demonstrates that a significant majority proportion of the Okanagan strains have unique inferred ancestry not seen elsewhere in the world, further bolstering the idea of the presence of Okanagan-specific $S$. uvarum population.

A phylogenetic tree was also created in order to visualize the genetic relatedness of the 102 indigenous Okanagan S. uvarum strains compared to the 12 international S. uvarum strains (Fig 6). These strains were differentiated into six sub-populations using the same $\mathrm{K}=5$ clustering output from InStruct that was used to visualize population structure in the DISTRUCT plots, which used Bayesian clustering to find inferred ancestry of the strains. Strains were coloured based on dominant inferred ancestry clustering, defined as representing at least $75 \%$ of the inferred ancestry profile based on the InStruct analysis for $\mathrm{K}=5$ clusters. Strains without a dominant inferred ancestor were left uncoloured (black). This clustering method identified two ancestors that appear unique to the Okanagan, and to each vintage: K3 (green) is unique to 2017, while K5 (orange) is unique to 2015. Additionally, the inferred ancestor K4 (purple) 


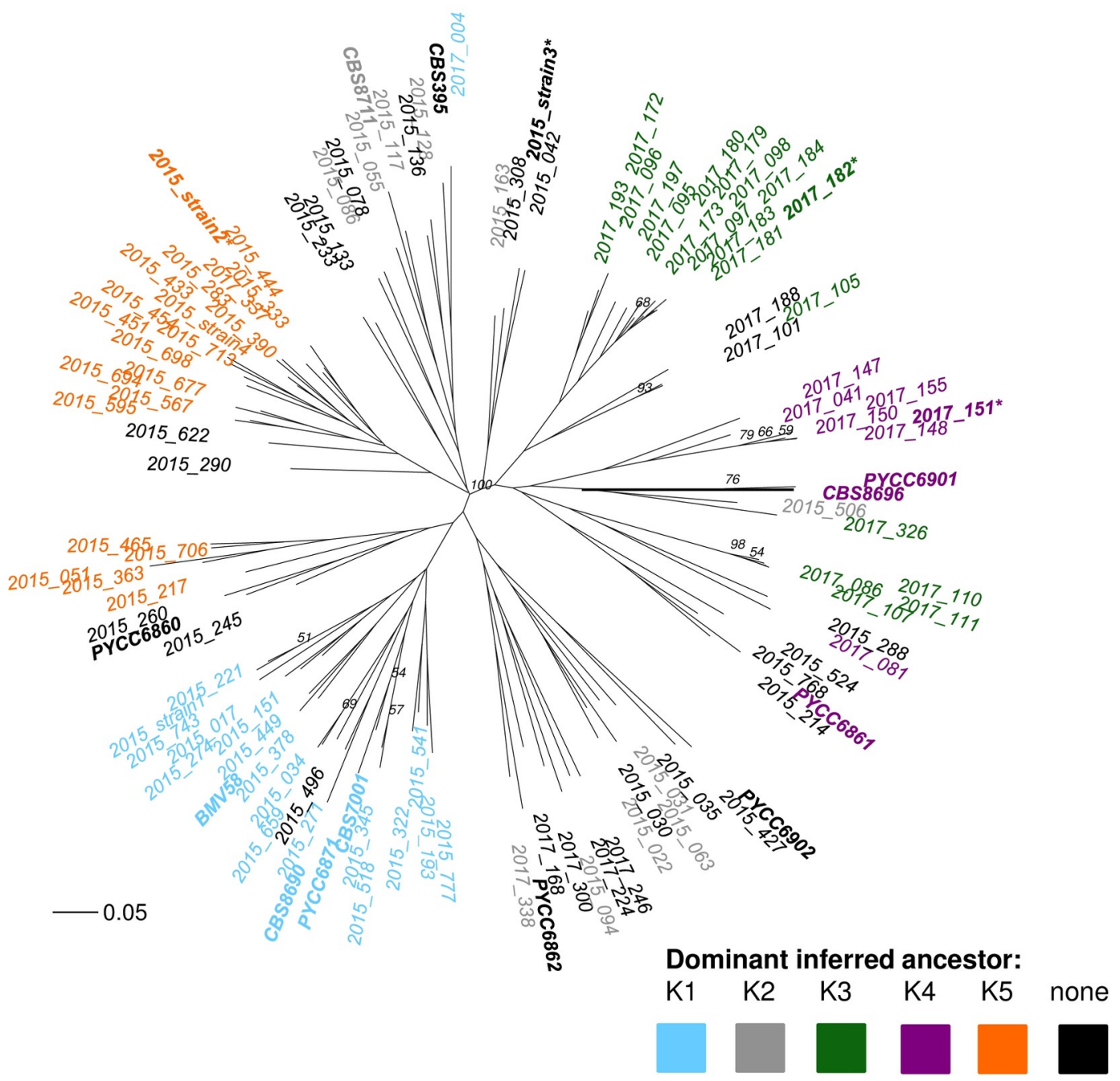

Fig 6. Phylogenetic tree of Okanagan and international S. uvarum strains. An unrooted, neighbour-joining phylogenetic tree using Bruvo distance, comparing the genetic relatedness of the $S$. uvarum strains identified in Chardonnay fermentations in the Okanagan Valley (Canada) during the 2017 vintage, as well as 12 selected S. uvarum strains isolated from around the world (see Table 2 for strain origins). The international strains are shown in bold, and the four dominant strains identified in this study are shown with an asterisk. Strains are coloured based on dominant inferred ancestry clustering, defined as representing at least $75 \%$ of the inferred ancestry profile based on an InStruct analysis for K $=5$ clusters. Strains without a dominant inferred ancestor were left uncoloured. A Bruvo distance of 0.05 is shown for scale. Bootstrap values above 0.5 are indicated.

https://doi.org/10.1371/journal.pone.0225615.g006

was identified as a Pacific west coast ancestor, because it contained Okanagan strains as well as strains from other Pacific west coast locations. The strains with K5 (orange) as their dominant inferred ancestor grouped together on the phylogenetic tree (Fig 6). Interestingly, the strains with K3 (green) or K4 (purple) as their dominant inferred ancestor grouped closely together, suggesting that these strains (most of which were identified in 2017) are genetically similar, and that these two inferred ancestors may have been closely related. The strains with K1 (blue), as their dominant inferred ancestor clustered together, separate from the other strains. This group contained Okanagan strains first identified during the 2015 vintage as well as international strains from Europe (Spain, Portugal, and Moldova). Finally, the strains with K2 (grey) as their dominant inferred ancestor grouped closely with the strains that had no dominant ancestor, and were spread over multiple branches of the phylogenetic tree. These strains 
had $\mathrm{K} 2$ (grey) as a dominant ancestor only in the $\mathrm{K}=5$ clustering method (Fig 5): in the $\mathrm{K}=11$ clustering method, these strains all contained multiple minor inferred ancestors (none of them dominant), which can explain why they are seen clustering with the strains that have no dominant inferred ancestry in this tree. The four dominant strains from this study (in bold and marked with an asterisk in Fig 6) all belonged to separate clusters, as was expected based on their inferred ancestry profiles (Fig 5).

The only commercial S. uvarum strain included in the construction of our phylogenetic tree was Velluto ${ }^{\mathbb{R}}$ BMV58 (Lallemand, Montreal, QC, Canada). Recently, one additional $S$. uvarum strain and one hybrid S. uvarum $\times$ S. cerevisiae strain have been commercially released: VitiFerm ${ }^{\mathrm{TM}}$ Sauvage BIO and EnartisFerm ${ }^{\mathbb{R}}$ ES U42, respectively. Some enological properties of $S$. uvarum have been studied, but more research is needed to allow winemakers to make informed decisions when selecting these non-traditional yeast strains for inoculation. S. uvarum produces lower levels of ethanol, acetic acid, and acetaldehyde, and higher levels of glycerol, succinic acid, malic acid, isoamyl alcohol, isobutanol, and ethyl acetate, as compared to S. cerevisiae [31-34]. Furthermore, due to its relatively lower production of ethanol, $S$. uvarum has been suggested as a potential means of mitigating the effects of climate change on winemaking [114]. With a warming climate, many winemaking regions are beginning to produce very ripe grapes with high sugar contents, which, with traditional fermentation techniques, can result in wines with very high ethanol contents. If this trend continues, many wines produced in the future could contain alcohol concentrations above the legal regulations of some countries, since diluting grape must with water is not permitted in wine production. Using non-traditional yeasts such as S. uvarum or non-Saccharomyces species, which can metabolize grape sugars into compounds other than ethanol (such as glycerol), may help mitigate this issue by keeping ethanol production within permitted levels. The recent increase in the availability of commercial S. uvarum strains indicates that this is clearly an area of interest for winemakers, and our changing climate and consumer preferences require investigation into new and creative methods of wine production, including the use of non-traditional yeasts such as $S$. uvarum, either alone or in combination with S. cerevisiae.

\section{Conclusions}

This study investigated the fungal communities and S. uvarum populations present in uninoculated commercial Chardonnay fermentations of grapes that originated from two different vineyards. Differences in fungal community composition were observed, with $H$. osmophila representing a larger proportion of the fungal community in the fermentations from one vineyard over the other. However, in all of the fermentations, S. uvarum was the dominant yeast during the early, mid, and late stages of alcoholic fermentation. An investigation into the genetic diversity of the S. uvarum strains present in this study was conducted, and this population was found to be both highly diverse and genetically distinct from S. uvarum strains identified in other regions of the world. A total of 106 S. uvarum strains were identified in this study, but only four strains played a dominant role in fermentation; two of these dominant strains were also identified as dominant strains at this same winery two years previously. The presence of persistent non-commercial strains, as well as the population structure analysis generated to compare Okanagan and international strains, provides evidence for an indigenous S. uvarum population with unique genotypes in the Okanagan Valley of British Columbia, Canada.

\section{Supporting information}

S1 Fig. Vineyard sampling map. Sampling layouts for (A) Vineyard 2 and (B) Vineyard 8. Each of the two conjoined squares represent the generated site of collection for one sample, 
with the sample number also given at each sampling site. One sampling site contains approximately 15 vines, and two grape clusters were taken from each vine (one on either side of the row), for a total of 30 clusters per sampling site. The geographic orientation of each vineyard is indicated in the bottom right corner of each sampling map.

(DOCX)

S2 Fig. Yeast isolate plating on Wallerstein Nutrient (WLN) media. (A) Forty-seven yeast colonies per barrel, per sampling stage, were isolated and plated onto WLN media in order to distinguish between presumed S. cerevisiae and presumed S. uvarum isolates prior to straintyping. On each plate, an S. cerevisiae control (Lalvin BA11) and an S. uvarum control (CBS 7001) were used to help aid the differentiation between the two species. Presumed S. cerevisiae isolates appeared cream-coloured, while presumed S. uvarum isolates appeared green. (B) Comparison of colony colour of a pure S. cerevisiae strain (Lalvin BA11), a pure S. uvarum strain (CBS 7001), and a S. cerevisiae $\mathrm{x}$ S. uvarum hybrid (Lalvin S6U), plated on WLN media. (DOCX)

S3 Fig. Histogram of pairwise genetic distances of multilocus genotypes analyzed in poppr (R package). The plot shows genetic distance cutoff as a function of the number of multilocus lineages, depending on the clustering method used.

(DOCX)

S4 Fig. Restriction analysis of yeast DNA using HaeIII. Restriction digest profiles of 50 yeast isolates, representing the 50 most abundant strains found in this study, as compared to reference strains: a pure S. cerevisiae strain (Fermol Mediterranée), a pure $S$. uvarum strain (CBS 7001), and a S. cerevisiae x S. uvarum hybrid strain (Lalvin S6U). (A) First 25 strains, as well as a ladder and all three reference strains. (B) Second 25 strains, as well as a ladder and all three reference strains.

(DOCX)

S5 Fig. Rarefaction curves. Rarefaction curves featuring (A) species richness in the fungal community, and (B) strain richness in the S. uvarum community, at different sampling depths. (DOCX)

S1 Table. Results of Levene's test for equal variance among groups for all chemical parameters measured. Any significant differences $(p \leq 0.05)$ are in bold. (DOCX)

S2 Table. Fungal community composition of Chardonnay grapes, must, and fermenting wine sourced from two different vineyards, based on 20,000 sequences per sample and represented as percent (\%) relative abundance. Samples were taken from grapes in the vineyard $(G)$, and at four stages of fermentation in the winey: cold settling (C), early (E), mid (M), and late (L). Vineyard 2 grape sample values are the means \pm SEM of five replicates, and Vineyard 8 grape sample values are the means \pm SEM of 6 replicates. All winery fermentation stages have three reported replicates, with the exception of the cold settling stage from the Vineyard 8 fermentations, which contained two. Sequences were identified to the species level unless otherwise indicated. Fungal species that represented less than $10 \%$ of the relative abundance in at least two samples were grouped into the Minor Fungi category. One exception was Saccharomyces cerevisiae, which never reached $10 \%$ relative abundance in any sample but is included in this table because of its importance during alcoholic fermentation. The last two columns indicate positive (Pos) and negative (Neg) controls. For the raw data containing all the fungi identified in this study (including minor fungi), please visit https://osf.io/j7rx8/.

(DOCX) 
S3 Table. Microsatellite identities of the representative multilocus genotypes (MLGs) of Saccharomyces uvarum strains isolated from stainless steel barrel-fermented Chardonnay at Canadian winery during the 2017 vintage. Allele sizes for allele 1 (A1) and allele 2 (A2) are shown for each of the 11 microsatellite loci analyzed. Strains with the prefix "2017" were isolated exclusively during the 2017 vintage. Strains with the prefix " 2015 ” were previously isolated and characterized during the 2015 vintage at the same winery, and were also isolated during the 2017 vintage. Strains without a vintage prefix are those that belong to global yeast databases (see S5 Table).

(DOCX)

S4 Table. Saccharomyces uvarum population composition of Chardonnay grapes, must, and fermenting wine sourced from two different vineyards, based on 32 yeast isolates per sample. Samples were taken at three stages of fermentation: early (E), mid (M), and late (L). Values are the means $\pm \operatorname{SEM}(n=3)$. Strains that represented less than $10 \%$ of the relative abundance in at least two samples were grouped into the Minor Strains category. For the raw data, containing all the S. uvarum strains identified in this study (including minor strains), please visit https://osf.io/j7rx8/.

(DOCX)

S5 Table. Microsatellite identities of the multilocus genotypes (MLGs) of Saccharomyces uvarum strains obtained from global yeast databases. Allele sizes for allele 1 (A1) and allele 2 (A2) are shown for each of the 11 microsatellite loci.

(DOCX)

\section{Acknowledgments}

The authors would like to thank the winemakers Darryl Brooker, Corrie Krehbiel, and Alexandra Haselich of Mission Hill Family Estate Winery for their assistance, guidance, and donation of fermentation samples. We also thank Britney Johnston and Mehrbod Estaki of the University of British Columbia for technical support and high-throughput amplicon sequencing data analysis support, respectively.

\section{Author Contributions}

Conceptualization: Garrett C. McCarthy, Jonathan T. Martiniuk, Vivien Measday, Daniel M. Durall.

Data curation: Garrett C. McCarthy, Sydney C. Morgan, Jonathan T. Martiniuk, Brianne L. Newman.

Formal analysis: Garrett C. McCarthy, Sydney C. Morgan.

Funding acquisition: Vivien Measday, Daniel M. Durall.

Investigation: Garrett C. McCarthy, Sydney C. Morgan, Brianne L. Newman, Stephanie E. McCann.

Methodology: Garrett C. McCarthy, Sydney C. Morgan, Jonathan T. Martiniuk, Brianne L. Newman, Stephanie E. McCann.

Project administration: Vivien Measday, Daniel M. Durall.

Software: Sydney C. Morgan. 
Supervision: Vivien Measday, Daniel M. Durall.

Validation: Garrett C. McCarthy, Sydney C. Morgan.

Visualization: Garrett C. McCarthy, Sydney C. Morgan.

Writing - original draft: Garrett C. McCarthy, Sydney C. Morgan.

Writing - review \& editing: Garrett C. McCarthy, Sydney C. Morgan, Jonathan T. Martiniuk, Brianne L. Newman, Stephanie E. McCann, Vivien Measday, Daniel M. Durall.

\section{References}

1. Suárez-Lepe JA, Morata A. New trends in yeast selection for winemaking. Trends Food Sci Technol. 2012; 23: 39-50. https://doi.org/10.1016/j.tifs.2011.08.005

2. Albertin W, Zimmer A, Miot-Sertier C, Bernard M, Coulon J, Moine V, et al. Combined effect of the Saccharomyces cerevisiae lag phase and the non-Saccharomyces consortium to enhance wine fruitiness and complexity. Appl Microbiol Biotechnol. 2017; 101: 7603-7620. https://doi.org/10.1007/s00253017-8492-1 PMID: 28913648

3. Capozzi V, Garofalo C, Chiriatti MA, Grieco F, Spano G. Microbial terroir and food innovation: The case of yeast biodiversity in wine. Microbiol Res. 2015; 181: 75-83. https://doi.org/10.1016/j.micres. 2015.10.005 PMID: 26521127

4. Blanco $P$, Orriols I, Losada A. Survival of commercial yeasts in the winery environment and their prevalence during spontaneous fermentations. J Ind Microbiol Biotechnol. 2011; 38: 235-239. https://doi. org/10.1007/s10295-010-0818-2 PMID: 20803347

5. Clavijo A, Calderón IL, Paneque P. Effect of the use of commercial Saccharomyces strains in a newly established winery in Ronda (Málaga, Spain). Antonie van Leeuwenhoek, Int J Gen Mol Microbiol. 2011; 99: 727-731. https://doi.org/10.1007/s10482-010-9514-5 PMID: 20886291

6. Morgan SC, Scholl CM, Benson NL, Stone ML, Durall DM. Sulfur dioxide addition at crush alters Saccharomyces cerevisiae strain composition in spontaneous fermentations at two Canadian wineries. Int J Food Microbiol. 2017; 244: 96-102. https://doi.org/10.1016/j.ijfoodmicro.2016.12.025 PMID: 28086153

7. Scholl CM, Morgan SC, Stone ML, Tantikachornkiat M, Neuner M, Durall DM. Composition of Saccharomyces cerevisiae strains in spontaneous fermentations of Pinot Noir and Chardonnay. Aust J Grape Wine Res. 2016; 22: 384-390. https://doi.org/10.1111/ajgw.12221

8. Constantí M, Poblet M, Arola L, Mas A, Guillamón JM. Analysis of yeast population during alcoholic fermentation in a newly established winery. Am J Enol Vitic. 1997; 48: 339-343.

9. Knight S, Klaere S, Fedrizzi B, Goddard MR. Regional microbial signatures positively correlate with differential wine phenotypes: Evidence for a microbial aspect to terroir. Sci Rep. 2015; 5: 14233. https:// doi.org/10.1038/srep14233 PMID: 26400688

10. Ciani M, Mannazzu I, Marinangeli P, Clementi F, Martini A. Contribution of winery-resident Saccharomyces cerevisiae strains to spontaneous grape must fermentation. Antonie Van Leeuwenhoek. 2004; 85: 159-164. https://doi.org/10.1023/B:ANTO.0000020284.05802.d7 PMID: 15031657

11. Santamaría $P$, López R, López E, Garijo $P$, Gutiérrez AR. Permanence of yeast inocula in the winery ecosystem and presence in spontaneous fermentations. Eur Food Res Technol. 2008; 227: 15631567. https://doi.org/10.1007/s00217-008-0855-5

12. Demuyter C, Lollier M, Legras J-L, Le Jeune C. Predominance of Saccharomyces uvarum during spontaneous alcoholic fermentation, for three consecutive years, in an Alsatian winery. J Appl Microbiol. 2004; 97: 1140-1148. https://doi.org/10.1111/j.1365-2672.2004.02394.x PMID: 15546404

13. Naumov GI, Masneuf I, Naumova ES, Aigle M, Dubourdieu D. Association of Saccharomyces bayanus var. uvarum with some French wines: Genetic analysis of yeast populations. Res Microbiol. 2000; 151: 683-691. https://doi.org/10.1016/s0923-2508(00)90131-1 PMID: 11081582

14. Naumov GI, Naumova ES, Antunovics Z, Sipiczki M. Saccharomyces bayanus var. uvarum in Tokaj wine-making of Slovakia and Hungary. Appl Microbiol Biotechnol. 2002; 59: 727-730. https://doi.org/ 10.1007/s00253-002-1077-6 PMID: 12226732

15. Morgan SC, McCarthy GC, Watters BS, Tantikachornkiat M, Zigg I, Cliff MA, et al. Effect of sulfite addition and pied de cuve inoculation on the microbial communities and sensory profiles of Chardonnay wines: dominance of indigenous Saccharomyces uvarum at a commercial winery. FEMS Yeast Res. 2019; 19: foz049. https://doi.org/10.1093/femsyr/foz049 PMID: 31344230 
16. Borneman AR, Pretorius IS. Genomic insights into the Saccharomyces sensu stricto complex. Genetics. 2015; 199: 281-291. https://doi.org/10.1534/genetics.114.173633 PMID: 25657346

17. Alsammar H, Delneri D. An update on the diversity, ecology and biogeography of the Saccharomyces genus. FEMS Yeast Res. 2020; 20: foaa013. https://doi.org/10.1093/femsyr/foaa013 PMID: 32196094

18. Almeida $\mathrm{P}$, Gonçalves $\mathrm{C}$, Teixeira $\mathrm{S}$, Libkind $\mathrm{D}$, Bontrager $\mathrm{M}$, Masneuf-Pomarède I, et al. A Gondwanan imprint on global diversity and domestication of wine and cider yeast Saccharomyces uvarum. Nat Commun. 2014; 5: 4044. https://doi.org/10.1038/ncomms5044 PMID: 24887054

19. Libkind D, Hittinger C, Valério E, Gonçalves C, Dover J, Johnston M, et al. Microbe domestication and the identification of the wild genetic stock of lager-brewing yeast. Proc Natl Acad Sci. 2011; 108 : 14539-14544. https://doi.org/10.1073/pnas.1105430108 PMID: 21873232

20. Sampaio JP, Gonçalves P. Natural populations of Saccharomyces kudriavzevii in Portugal are associated with oak bark and are sympatric with S. cerevisiae and S. paradoxus. Appl Environ Microbiol. 2008; 74: 2144-2152. https://doi.org/10.1128/AEM.02396-07 PMID: 18281431

21. Naumov GI, Nguyen H-V, Naumova ES, Michel A, Aigle M, Gaillardin C. Genetic identification of Saccharomyces bayanus var. uvarum, a cider-fermenting yeast. Int J Food Microbiol. 2001. Available: www.elsevier.nlrlocaterijfoodmicro https://doi.org/10.1016/s0168-1605(00)00515-8 PMID: 11393685

22. Masneuf-Pomarede I, Le Jeune C, Durrens P, Lollier M, Aigle M, Dubourdieu D. Molecular typing of wine yeast strains Saccharomyces bayanus var. uvarum using microsatellite markers. Syst Appl Microbiol. 2007; 30: 75-82. https://doi.org/10.1016/j.syapm.2006.02.006 PMID: 16621402

23. Coton E, Coton M, Levert D, Casaregola S, Sohier D. Yeast ecology in French cider and black olive natural fermentations. Int J Food Microbiol. 2006; 108: 130-135. https://doi.org/10.1016/j.ijfoodmicro. 2005.10.016 PMID: 16380183

24. Suárez Valles B, Pando Bedriñana R, Fernández Tascón N, Querol Simón A, Rodríguez Madrera R Yeast species associated with the spontaneous fermentation of cider. Food Microbiol. 2007; 24: 2531. https://doi.org/10.1016/j.fm.2006.04.001 PMID: 16943091

25. Fernández-Espinar MT, López V, Ramón D, Bartra E, Querol A. Study of the authenticity of commercial wine yeast strains by molecular techniques. Int J Food Microbiol. 2001; 70: 1-10. https://doi.org/ 10.1016/s0168-1605(01)00502-5 PMID: 11759747

26. Nguyen HV, Gaillardin C. Evolutionary relationships between the former species Saccharomyces uvarum and the hybrids Saccharomyces bayanus and Saccharomyces pastorianus; reinstatement of Saccharomyces uvarum (Beijerinck) as a distinct species. FEMS Yeast Res. 2005; 5: 471-483. https://doi.org/10.1016/j.femsyr.2004.12.004 PMID: 15691752

27. Rainieri S, Zambonelli C, Hallsworth JE, Pulvirenti A, Giudici P. Saccharomyces uvarum, a distinct group within Saccharomyces sensu stricto. FEMS Microbiol Lett. 1999; 177: 177-185. https://doi.org/ 10.1111/j.1574-6968.1999.tb13729.x PMID: 10436934

28. Zhang H, Richards KD, Wilson S, Lee SA, Sheehan H, Roncoroni M, et al. Genetic characterization of strains of Saccharomyces uvarum from New Zealand wineries. Food Microbiol. 2015; 46: 92-99. https://doi.org/10.1016/j.fm.2014.07.016 PMID: 25475271

29. Rodríguez ME, Pérez-Través L, Sangorrín MP, Barrio E, Lopes CA. Saccharomyces eubayanus and Saccharomyces uvarum associated with the fermentation of Araucaria araucana seeds in Patagonia. FEMS Yeast Res. 2014; 14: 948-965. https://doi.org/10.1111/1567-1364.12183 PMID: 25041507

30. Rodríguez M, Pérez-Través L, Sangorrín M, Barrio E, Querol A, Lopes C. Saccharomyces uvarum is responsible for the traditional fermentation of apple Chicha in Patagonia. FEMS Yeast Res. 2017; 17: fow109. https://doi.org/10.1093/femsyr/fow109 PMID: 28011906

31. Castellari L, Ferruzzi M, Magrini A, Giudici P, Passarelli $P$, Zambonelli C. Unbalanced wine fermentation by cryotolerant vs. non-cryotolerant Saccharomyces strains. Vitis. 1994; 33: 49-52. Available: https://www.vitis-vea.de/admin/volltext/e033350.pdf

32. Gamero A, Tronchoni J, Querol A, Belloch C. Production of aroma compounds by cryotolerant Saccharomyces species and hybrids at low and moderate fermentation temperatures. J Appl Microbiol. 2013; 114: 1405-1414. https://doi.org/10.1111/jam.12126 PMID: 23294204

33. Magyar I, Tóth T. Comparative evaluation of some oenological properties in wine strains of Candida stellata, Candida zemplinina, Saccharomyces uvarum and Saccharomyces cerevisiae. Food Microbiol. 2011; 28: 94-100. https://doi.org/10.1016/j.fm.2010.08.011 PMID: 21056780

34. Sipiczki M, Romano P, Lipani G, Miklos I, Antunovics Z. Analysis of yeasts derived from natural fermentation in a Tokaj winery. Antonie van Leeuwenhoek, Int J Gen Mol Microbiol. 2001; 79: 97-105 https://doi.org/10.1023/A:1010249408975 
35. Deed RC, Fedrizzi B, Gardner RC. Influence of fermentation temperature, yeast strain, and grape juice on the aroma chemistry and sensory profile of Sauvignon blanc wines. J Agric Food Chem. 2017; 65: 8902-8912. https://doi.org/10.1021/acs.jafc.7b03229 PMID: 28922915

36. Castelluci F. Microbiological analysis of wines and musts Method OIV-MA-AS4-01 Type IV Method. Compendium of International Methods of Analysis-OIV. OIV; 2010. pp. 1-32.

37. Houck L. Use of biphenyl for reducing Penicillium decay of stored citrus. Agric Res Serv. 1971.

38. Martiniuk JT, Pacheco B, Russell G, Tong S, Backstrom I, Measday V. Impact of commercial strain use on Saccharomyces cerevisiae population structure and dynamics in Pinot noir vineyards and spontaneous fermentations of a Canadian winery. PLoS One. 2016; 11: e0160259. https://doi.org/10. 1371/journal.pone.0160259 PMID: 27551920

39. Zott K, Miot-Sertier C, Claisse O, Lonvaud-Funel A, Masneuf-Pomarede I. Dynamics and diversity of non-Saccharomyces yeasts during the early stages in winemaking. Int J Food Microbiol. 2008; 125 : 197-203. https://doi.org/10.1016/j.ijfoodmicro.2008.04.001 PMID: 18495281

40. Mortimer R, Polsinelli M. On the origins of wine yeast. Res Microbiol. 1999; 150: 199-204. https://doi. org/10.1016/s0923-2508(99)80036-9 PMID: 10229949

41. Masneuf-Pomarede I, Salin F, Börlin M, Coton E, Coton M, Le Jeune C, et al. Microsatellite analysis of Saccharomyces uvarum diversity. FEMS Yeast Res. 2016; 16: fow002. https://doi.org/10.1093/ femsyr/fow002 PMID: 26772797

42. Bruvo R, Michiels NK, D'Souza TG, Schulenburg H. A simple method for the calculation of microsatellite genotype distances irrespective of ploidy level. Mol Ecol. 2004; 13: 2101-2106. https://doi.org/10. 1111/j.1365-294X.2004.02209.x PMID: 15189230

43. Kamvar ZN, Brooks JC, Grünwald NJ. Novel R tools for analysis of genome-wide population genetic data with emphasis on clonality. Front Genet. 2015; 6: 208. https://doi.org/10.3389/fgene.2015.00208 PMID: 26113860

44. Kamvar ZN, Grünwald NJ. Analysis of multilocus genotypes and lineages in poppr 2.8.6.99.18. In: GitHub [Internet]. 2020 [cited 29 Oct 2020]. Available: https://grunwaldlab.github.io/poppr/articles/mlg. html\#choosing-a-threshold\%0AAnalysis

45. Sebastiani F, Barberio C, Casalone E, Cavalieri D, Polsinelli M. Crosses between Saccharomyces cerevisiae and Saccharomyces bayanus generate fertile hybrids. Res Microbiol. 2002; 153: 53-58. https://doi.org/10.1016/s0923-2508(01)01286-4 PMID: 11881899

46. Zott K, Claisse O, Lucas P, Coulon J, Lonvaud-Funel A, Masneuf-Pomarede I. Characterization of the yeast ecosystem in grape must and wine using real-time PCR. Food Microbiol. 2010; 27: 559-567. https://doi.org/10.1016/j.fm.2010.01.006 PMID: 20510771

47. Morgan SC, Tantikachornkiat M, Scholl CM, Benson NL, Cliff MA, Durall DM. The effect of sulfur dioxide addition at crush on the fungal and bacterial communities and the sensory attributes of Pinot gris wines. Int J Food Microbiol. 2019; 290: 1-14. https://doi.org/10.1016/j.ijfoodmicro.2018.09.020 PMID: 30278370

48. Bokulich NA, Mills DA. Improved selection of Internal Transcribed Spacer-specific primers enables quantitative, ultra-high-throughput profiling of fungal communities. Appl Environ Microbiol. 2013; 79: 2519-2526. https://doi.org/10.1128/AEM.03870-12 PMID: 23377949

49. Bolyen E, Rideout JR, Dillon MR, Bokulich NA, Abnet CC, Al-Ghalith GA, et al. Reproducible, interactive, scalable and extensible microbiome data science using QIIME 2. Nat Biotechnol. 2019;7. https:// doi.org/10.1038/nbt0119-7 PMID: 30605161

50. Callahan BJ, McMurdie PJ, Rosen MJ, Han AW, Johnson AJA, Holmes SP. DADA2: High resolution sample inference from Illumina amplicon data. Nat Methods. 2016; 13: 581-583. https://doi.org/10. 1038/nmeth.3869 PMID: 27214047

51. Morgan M, Anders S, Lawrence M, Aboyoun P, Pagès H, Gentleman R. ShortRead: A bioconductor package for input, quality assessment and exploration of high-throughput sequence data. Bioinformatics. 2009; 25: 2607-2608. https://doi.org/10.1093/bioinformatics/btp450 PMID: 19654119

52. Martin M. Cutadapt removes adapter sequences from high-throughput sequencing reads. EMBnet. journal. 2011; 17: 10-12. https://doi.org/10.14806/ej.17.1.200

53. Blaalid R, Kumar S, Nilsson RH, Abarenkov K, Kirk PM, Kauserud H. ITS1 versus ITS2 as DNA metabarcodes for fungi. Mol Ecol Resour. 2013; 13: 218-224. https://doi.org/10.1111/1755-0998.12065 PMID: 23350562

54. Feibelman $T$, Bayman $P$, Cibula WG. Length variation in the internal transcribed spacer of ribosomal DNA in chanterelles. Mycol Res. 1994; 98: 614-618. https://doi.org/10.1016/S0953-7562(09)80407-3

55. Katoh K, Standley DM. MAFFT multiple sequence alignment software version 7: Improvements in performance and usability. Mol Biol Evol. 2013; 30: 772-780. https://doi.org/10.1093/molbev/mst010 PMID: 23329690 
56. Price MN, Dehal PS, Arkin AP. FastTree 2-Approximately Maximum-Likelihood Trees for Large Alignments. Poon AFY, editor. PLoS One. 2010; 5: e9490. https://doi.org/10.1371/journal.pone.0009490 PMID: 20224823

57. Kõljalg U, Nilsson RH, Abarenkov K, Tedersoo L, Taylor AFS, Bahram M, et al. Towards a unified paradigm for sequence-based identification of fungi. Mol Ecol. 2013; 22: 5271-5277. https://doi.org/10. 1111/mec.12481 PMID: 24112409

58. Blanca MJ, Alarcón R, Arnau J, Bono R, Bendayan R. Non-normal data: is ANOVA still a valid option? Psicothema. 2017; 29: 552-557. https://doi.org/10.7334/psicothema2016.383 PMID: 29048317

59. Oksanen J, Blanchet FGB, Friendly M, Kindt R, Legendre P, McGlinn D, et al. vegan: Community Ecology Package. R; 2018. Available: https://cran.r-project.org/package=vegan

60. Anderson MJ, Walsh DCI. PERMANOVA, ANOSIM, and the Mantel test in the face of heterogeneous dispersions: What null hypothesis are you testing? Ecol Monogr. 2013; 83: 557-574. https://doi.org/ 10.1890/12-2010.1

61. Lorion CM, Kennedy BP. Relationships between deforestation, riparian forest buffers and benthic macroinvertebrates in neotropical headwater streams. Freshw Biol. 2009; 54: 165-180. https://doi. org/10.1111/j.1365-2427.2008.02092.x

62. Gao H, Williamson S, Bustamante CD. A Markov Chain Monte Carlo Approach for Joint Inference of Population Structure and Inbreeding Rates From Multilocus Genotype Data. Genetics. 2007; 176: 1635-1651. https://doi.org/10.1534/genetics.107.072371 PMID: 17483417

63. Gao H, Bryc K, Bustamante CD. On Identifying the Optimal Number of Population Clusters via the Deviance Information Criterion. PLoS One. 2011; 6: 21014. https://doi.org/10.1371/journal.pone. 0021014 PMID: 21738600

64. Jakobsson M, Rosenberg NA. Genetics and population analysis CLUMPP: a cluster matching and permutation program for dealing with label switching and multimodality in analysis of population structure. 2007; 23: 1801-1806. https://doi.org/10.1093/bioinformatics/btm233 PMID: 17485429

65. Rosenberg NA. distruct: a program for the graphical display of population structure. Mol Ecol Notes. 2003; 4: 137-138. https://doi.org/10.1046/j.1471-8286.2003.00566.x

66. Gayevskiy V, Klaere S, Knight S, Goddard MR. ObStruct: A method to objectively analyse factors driving population structure using Bayesian ancestry profiles. PLoS One. 2014; 9: e85196. https://doi.org/ 10.1371/journal.pone.0085196 PMID: 24416362

67. Peakall R, Smouse PE. GenAIEx 6.5: Genetic analysis in Excel. Population genetic software for teaching and research—an update. Bioinformatics. 2012; 28: 2537-2539. https://doi.org/10.1093/ bioinformatics/bts460 PMID: 22820204

68. Peakall R, Smouse PE. GENALEX 6: genetic analysis in Excel. Population genetic software for teaching and research. Mol Ecol Notes. 2006; 6: 288-295. https://doi.org/10.1111/j.1471-8286.2005.01155.x

69. Paradis E, Schliep K. ape 5.0: an environment for modern phylogenetics and evolutionary analyses in R. Schwartz R, editor. Bioinformatics. 2019; 35: 526-528. https://doi.org/10.1093/bioinformatics/ bty633 PMID: 30016406

70. Amerine MA, Roessler EB. Wines: Their Sensory Evaluation. New York: W.H.Freeman \& Co Ltd; 1983.

71. Wang C, Mas A, Esteve-Zarzoso B. The interaction between Saccharomyces cerevisiae and non-Saccharomyces yeast during alcoholic fermentation is species and strain specific. Front Microbiol. 2016; 7: 1-11. https://doi.org/10.3389/fmicb.2016.00001 PMID: 26834723

72. Varela C, Borneman AR. Yeasts found in vineyards and wineries. Yeast. 2017; 34: 111-128. https:// doi.org/10.1002/yea.3219 PMID: 27813152

73. Takahashi M, Ohta T, Masaki K, Mizuno A, Goto-Yamamoto N. Evaluation of microbial diversity in sulfite-added and sulfite-free wine by culture-dependent and -independent methods. J Biosci Bioeng. 2014; 117: 569-75. https://doi.org/10.1016/j.jbiosc.2013.10.012 PMID: 24239025

74. González V, Tello ML. The endophytic mycota associated with Vitis vinifera in central Spain. Fungal Divers. 2011; 47: 29-42. https://doi.org/10.1007/s13225-010-0073-x

75. Pancher M, Ceol M, Corneo PE, Longa CMO, Yousaf S, Pertot I, et al. Fungal endophytic communities in grapevines (Vitis vinifera L.) Respond to crop management. Appl Environ Microbiol. 2012; 78 : 4308-4317. https://doi.org/10.1128/AEM.07655-11 PMID: 22492448

76. Varanda CMR, Oliveira M, Materatski P, Landum M, Clara MIE, Félix M do R. Fungal endophytic communities associated to the phyllosphere of grapevine cultivars under different types of management. Fungal Biol. 2016; 120: 1525-1536. https://doi.org/10.1016/j.funbio.2016.08.002 PMID: 27890088

77. Martini M, Musetti R, Grisan S, Polizzotto R, Borselli S, Pavan F, et al. DNA-dependent detection of the grapevine fungal endophytes Aureobasidium pullulans and Epicoccum nigrum. Plant Dis. 2009; 93: 993-998. https://doi.org/10.1094/PDIS-93-10-0993 PMID: 30754374 
78. Kecskeméti E, Berkelmann-Löhnertz B, Reineke A. Are epiphytic microbial communities in the carposphere of ripening grape clusters (Vitis vinifera L.) different between conventional, organic, and biodynamic grapes? PLoS One. 2016;11. https://doi.org/10.1371/journal.pone.0160852 PMID: 27500633

79. Castañeda LE, Miura T, Sánchez R, Barbosa O. Effects of agricultural management on phyllosphere fungal diversity in vineyards and the association with adjacent native forests. PeerJ. 2018; 6: e5715. https://doi.org/10.7717/peerj.5715 PMID: 30397540

80. Bokulich NA, Thorngate JH, Richardson PM, Mills DA. Microbial biogeography of wine grapes is conditioned by cultivar, vintage, and climate. Proc Natl Acad Sci. 2014; 111: E139-E148. https://doi.org/10. 1073/pnas.1317377110 PMID: 24277822

81. Setati ME, Jacobson D, Bauer FF. Sequence-based analysis of the Vitis vinifera L. cv Cabernet sauvignon grape must mycobiome in three South African vineyards employing distinct agronomic systems. Front Microbiol. 2015; 6: 1358. https://doi.org/10.3389/fmicb.2015.01358 PMID: 26648930

82. Freire L, Passamani FRF, Thomas AB, Nassur R de CMR, Silva LM, Paschoal FN, et al. Influence of physical and chemical characteristics of wine grapes on the incidence of Penicillium and Aspergillus fungi in grapes and ochratoxin A in wines. Int J Food Microbiol. 2017; 241: 181-190. https://doi.org/10. 1016/j.ijfoodmicro.2016.10.027 PMID: 27792949

83. Inoue $\mathrm{T}$, Nagatomi $\mathrm{Y}$, Uyama A, Mochizuki N. Degradation of aflatoxin $\mathrm{B} 1$ during the fermentation of alcoholic beverages. Toxins (Basel). 2013; 5: 1219-1229. https://doi.org/10.3390/toxins5071219 PMID: 23812408

84. Mateo R, Medina Á, Mateo EM, Mateo F, Jiménez M. An overview of ochratoxin A in beer and wine. Int J Food Microbiol. 2007; 119: 79-83. https://doi.org/10.1016/j.ijfoodmicro.2007.07.029 PMID: 17716764

85. Soleas GJ, Yan J, Goldberg DM. Assay of ochratoxin A in wine and beer by high-pressure liquid chromatography photodiode array and gas chromatography mass selective detection. J Agric Food Chem. 2001; 49: 2733-2740. https://doi.org/10.1021/jf0100651 PMID: 11409959

86. Petruzzi L, Sinigaglia M, Corbo MR, Campaniello D, Speranza B, Bevilacqua A. Decontamination of ochratoxin A by yeasts: Possible approaches and factors leading to toxin removal in wine. Appl Microbiol Biotechnol. 2014; 98: 6555-6567. https://doi.org/10.1007/s00253-014-5814-4 PMID: 24841121

87. Brewer MT, Milgroom MG. Phylogeography and population structure of the grape powdery mildew fungus, Erysiphe necator, from diverse Vitis species. BMC Evol Biol. 2010; 10: 268. https://doi.org/10. 1186/1471-2148-10-268 PMID: 20809968

88. Gadoury DM, Seem RC, Wilcox WF, Henick-Kling T, Conterno L, Day A, et al. Effects of diffuse colonization of grape berries by Uncinula necator on bunch rots, berry microflora, and juice and wine quality. Phytopathology. 2007; 97: 1356-1365. https://doi.org/10.1094/PHYTO-97-10-1356 PMID: 18943695

89. Barata A, Malfeito-Ferreira M, Loureiro V. The microbial ecology of wine grape berries. Int J Food Microbiol. 2012; 153: 243-259. https://doi.org/10.1016/j.jifoodmicro.2011.11.025 PMID: 22189021

90. Combina M, Elía A, Mercado L, Catania C, Ganga A, Martinez C. Dynamics of indigenous yeast populations during spontaneous fermentation of wines from Mendoza, Argentina. Int J Food Microbiol. 2005; 99: 237-243. https://doi.org/10.1016/j.ijfoodmicro.2004.08.017 PMID: 15808358

91. Ocón E, Gutiérrez a. R, Garijo P, Tenorio C, López I, López R, et al. Quantitative and qualitative analysis of non-Saccharomyces yeasts in spontaneous alcoholic fermentations. Eur Food Res Technol. 2010; 230: 885-891. https://doi.org/10.1007/s00217-010-1233-7

92. Granchi L, Ganucci D, Messini A, Vincenzini M. Oenological properties of Hanseniaspora osmophila and Kloeckera corticis from wines produced by spontaneous fermentations of normal and dried grapes. FEMS Yeast Res. 2002; 2: 403-407. https://doi.org/10.1016/S1567-1356(02)00089-2 PMID: 12702291

93. Pateraki C, Paramithiotis S, Doulgeraki Al, Kallithraka S, Kotseridis Y, Drosinos EH. Effect of sulfur dioxide addition in wild yeast population dynamics and polyphenolic composition during spontaneous red wine fermentation from Vitis vinifera cultivar Agiorgitiko. Eur Food Res Technol. 2014; 239: 10671075. https://doi.org/10.1007/s00217-014-2303-z

94. Stefanini I, Albanese D, Cavazza A, Franciosi E, De Filippo C, Donati C, et al. Dynamic changes in microbiota and mycobiota during spontaneous "Vino Santo Trentino" fermentation. Microb Biotechnol. 2016; 9: 195-208. https://doi.org/10.1111/1751-7915.12337 PMID: 26780037

95. Bokulich NA, Swadener M, Sakamoto K, Mills DA, Bisson LF. Sulfur dioxide treatment alters wine microbial diversity and fermentation progression in a dose-dependent fashion. Am J Enol Vitic. 2014; 66: 1-21. https://doi.org/10.5344/ajev.2014.14096

96. Viana F, Gil J V., Genovés S, Vallés S, Manzanares P. Rational selection of non-Saccharomyces wine yeasts for mixed starters based on ester formation and enological traits. Food Microbiol. 2008; 25: 778-785. https://doi.org/10.1016/j.fm.2008.04.015 PMID: 18620969 
97. Ciani M, Comitini F, Mannazzu I, Domizio P. Controlled mixed culture fermentation: A new perspective on the use of non-Saccharomyces yeasts in winemaking. FEMS Yeast Res. 2010; 10: 123-133. https://doi.org/10.1111/j.1567-1364.2009.00579.x PMID: 19807789

98. Valero E, Cambon B, Schuller D, Casal M, Dequin S. Biodiversity of Saccharomyces yeast strains from grape berries of wine-producing areas using starter commercial yeasts. FEMS Yeast Res. 2007; 7: 317-329. https://doi.org/10.1111/j.1567-1364.2006.00161.x PMID: 17040482

99. Le Jeune C, Erny C, Demuyter C, Lollier M. Evolution of the population of Saccharomyces cerevisiae from grape to wine in a spontaneous fermentation. Food Microbiol. 2006; 23: 709-716. https://doi.org/ 10.1016/j.fm.2006.02.007 PMID: 16943073

100. Sturm J, Grossmann M, Schnell S. Influence of grape treatment on the wine yeast populations isolated from spontaneous fermentations. J Appl Microbiol. 2006; 101: 1241-1248. https://doi.org/10.1111/j. 1365-2672.2006.03034.x PMID: 17105554

101. Grangeteau C, Gerhards D, von Wallbrunn C, Alexandre H, Rousseaux S, Guilloux-Benatier M. Persistence of Two Non-Saccharomyces Yeasts (Hanseniaspora and Starmerella) in the Cellar. Front Microbiol. 2016; 7: 268. https://doi.org/10.3389/fmicb.2016.00268 PMID: 27014199

102. Pérez-Martín F, Seseña S, Fernández-González M, Arévalo M, Palop ML. Microbial communities in air and wine of a winery at two consecutive vintages. Int J Food Microbiol. 2014; 190: 44-53. https:// doi.org/10.1016/j.ijfoodmicro.2014.08.020 PMID: 25173918

103. Bokulich NA, Ohta M, Richardson PM, Mills DA. Monitoring seasonal changes in winery-resident microbiota. PLoS One. 2013; 8: e66437. https://doi.org/10.1371/journal.pone.0066437 PMID: 23840468

104. Santamaría P, Garijo P, López R, Tenorio C, Gutiérrez AR. Analysis of yeast population during spontaneous alcoholic fermentation: Effect of the age of the cellar and the practice of inoculation. Int $\mathrm{J}$ Food Microbiol. 2005; 103: 49-56. https://doi.org/10.1016/j.ijfoodmicro.2004.11.024 PMID: 16084265

105. Dellaglio F, Zapparoli G, Malacrinò P, Suzzi G, Torriani S. Saccharomyces bayanus var. uvarum and Saccharomyces cerevisiae succession during spontaneous fermentations of Recioto and Amarone wines. Ann Microbiol. 2003; 53: 411-425. Available: http://citeseerx.ist.psu.edu/viewdoc/download? doi=10.1.1.576.4605\&rep=rep1\&type=pdf

106. Csoma H, Zakany N, Capece A, Romano P, Sipiczki M. Biological diversity of Saccharomyces yeasts of spontaneously fermenting wines in four wine regions: Comparative genotypic and phenotypic analysis. Int J Food Microbiol. 2010; 140: 239-248. https://doi.org/10.1016/j.ijfoodmicro.2010.03.024 PMID: 20413169

107. Schütz M, Gafner J. Lower fructose uptake capacity of genetically characterized strains of Saccharomyces bayanus compared to strains of Saccharomyces cerevisiae: a likely cause of reduced alcoholic fermentation activity. Am J Enol Vitic. 1995; 46: 175-180.

108. Le Jeune C, Lollier M, Demuyter C, Erny C, Legras J-L, Aigle M, et al. Characterization of natural hybrids of Saccharomyces cerevisiae and Saccharomyces bayanus var. uvarum. FEMS Yeast Res. 2007; 7: 540-549. https://doi.org/10.1111/j.1567-1364.2007.00207.x PMID: 17302940

109. Sabate J, Cano J, Querol A, Guillamon JM. Diversity of Saccharomyces strains in wine fermentations: analysis for two consecutive years. Lett Appl Microbiol. 1998; 26: 452-455. https://doi.org/10.1046/j. 1472-765x.1998.00369.x PMID: 9717318

110. Tosi E, Azzolini M, Guzzo F, Zapparoli G. Evidence of different fermentation behaviours of two indigenous strains of Saccharomyces cerevisiae and Saccharomyces uvarum isolated from Amarone wine. J Appl Microbiol. 2009; 107: 210-218. https://doi.org/10.1111/j.1365-2672.2009.04196.x PMID: 19245401

111. Blanco P, Mirás-Avalos JM, Orriols I. Effect of must characteristics on the diversity of Saccharomyces strains and their prevalence in spontaneous fermentations. J Appl Microbiol. 2012; 112: 936-944. https://doi.org/10.1111/j.1365-2672.2012.05278.x PMID: 22394241

112. Stefanini I, Dapporto L, Legras J-L, Calabretta A, Di Paola M, De Filippo C, et al. Role of social wasps in Saccharomyces cerevisiae ecology and evolution. Proc Natl Acad Sci. 2012; 109: 13398-13403. https://doi.org/10.1073/pnas.1208362109 PMID: 22847440

113. Gayevskiy V, Goddard MR. Geographic delineations of yeast communities and populations associated with vines and wines in New Zealand. Int Soc Microb Ecol J. 2012; 6: 1281-1290. https://doi.org/ 10.1038/ismej.2011.195 PMID: 22189497

114. Alonso-del-Real J, Lairón-Peris M, Barrio E, Querol A. Effect of temperature on the prevalence of Saccharomyces non cerevisiae species against a S. cerevisiae wine strain in wine fermentation: competition, physiological fitness, and influence in final wine composition. Front Microbiol. 2017; 8: 1-15. https://doi.org/10.3389/fmicb.2017.00001 PMID: 28197127 